\title{
The adjacency matroid of a graph
}

\author{
Robert Brijder \\ Hasselt University and Transnational University of Limburg \\ Belgium \\ robert.brijder@uhasselt.be \\ Hendrik Jan Hoogeboom \\ LIACS, Leiden University \\ The Netherlands \\ h.j.hoogeboom@cs.leidenuniv.nl \\ Lorenzo Traldi \\ Lafayette College \\ Easton, Pennsylvania 18042 USA \\ traldil@lafayette.edu
}

Submitted: Nov 20, 2012; Accepted: Aug 17, 2013; Published: Aug 30, 2013

Mathematics Subject Classifications: 05C50

\begin{abstract}
If $G$ is a looped graph, then its adjacency matrix represents a binary matroid $M_{A}(G)$ on $V(G)$. $M_{A}(G)$ may be obtained from the delta-matroid represented by the adjacency matrix of $G$, but $M_{A}(G)$ is less sensitive to the structure of $G$. Jaeger proved that every binary matroid is $M_{A}(G)$ for some $G[$ Ann. Discrete Math. 17 (1983), 371-376].

The relationship between the matroidal structure of $M_{A}(G)$ and the graphical structure of $G$ has many interesting features. For instance, the matroid minors $M_{A}(G)-v$ and $M_{A}(G) / v$ are both of the form $M_{A}\left(G^{\prime}-v\right)$ where $G^{\prime}$ may be obtained from $G$ using local complementation. In addition, matroidal considerations lead to a principal vertex tripartition, distinct from the principal edge tripartition of Rosenstiehl and Read [Ann. Discrete Math. 3 (1978), 195-226]. Several of these results are given two very different proofs, the first involving linear algebra and the second involving set systems or $\Delta$-matroids. Also, the Tutte polynomials of the adjacency matroids of $G$ and its full subgraphs are closely connected to the interlace polynomial of Arratia, Bollobás and Sorkin [Combinatorica 24 (2004), 567-584].
\end{abstract}

Keywords: adjacency, delta-matroid, interlace polynomial, local complement, matroid, minor, Tutte polynomial

\section{Introduction}

A distinctive feature of matroid theory is that there are so many equivalent ways to define matroids, each providing its own special insight into the nature of the structure 
being defined. We refer to the books of Oxley [26], Welsh [35] and White [36, 37, 38] for thorough discussions. Here is one way to define a particular kind of matroid:

Definition 1. A binary matroid $M$ is an ordered pair $(V, \mathcal{C}(M))$, which satisfies the following circuit axioms:

1. $V$ is a finite set and $\mathcal{C}(M) \subseteq 2^{V}$.

2. $\varnothing \notin \mathcal{C}(M)$.

3. If $C_{1}, C_{2} \in \mathcal{C}(M)$ then $C_{1} \nsubseteq C_{2}$.

4. If $C_{1} \neq C_{2} \in \mathcal{C}(M)$ then the symmetric difference $\left(C_{1} \backslash C_{2}\right) \cup\left(C_{2} \backslash C_{1}\right)=C_{1} \Delta C_{2}$ contains at least one $C \in \mathcal{C}(M)$.

If $M$ and $M^{\prime}$ are matroids on $V$ and $V^{\prime}$ then $M \cong M^{\prime}$ if there is a bijection between $V$ and $V^{\prime}$ under which $\mathcal{C}(M)$ and $\mathcal{C}\left(M^{\prime}\right)$ correspond.

We consider $2^{V}$ as a vector space over $G F(2)$ in the usual way: if $S, S_{1}$ and $S_{2}$ are subsets of $V$ then $0 \cdot S=\varnothing, 1 \cdot S=S$ and $S_{1}+S_{2}=S_{1} \Delta S_{2}$.

Definition 2. If $M$ is a binary matroid on $V$ then the cycle space $Z(M)$ is the subspace of $2^{V}$ spanned by $\mathcal{C}(M)$.

The importance of the cycle space of a binary matroid is reflected in the well known fact that two fundamental ideas of matroid theory, nullity and duality, correspond under $Z$ to two fundamental ideas of linear algebra, dimension and orthogonality: $\operatorname{dim} Z(M)$ is the nullity of $M$, and if $M^{*}$ is the dual of $M$ then $Z\left(M^{*}\right)$ is the orthogonal complement of $Z(M)$. (See $[26,35,37]$ for details.) Ghouila-Houri [19] showed that the importance of the cycle space is reflected in another special property, mentioned by some authors $[8,22,37]$ but not stated explicitly in most accounts of the theory.

Theorem 3. [19] Let $V$ be a finite set. Then the function

$$
Z:\{\text { binary matroids on } V\} \rightarrow\left\{G F(2) \text {-subspaces of } 2^{V}\right\}
$$

is bijective.

Theorem 3 tells us that any construction or function which assigns a subspace of $2^{\mathrm{V}}$ to some object may be unambiguously reinterpreted as assigning a binary matroid to that object. There are of course many notions of linear algebra that involve assigning subspaces to objects. For instance, an $m \times n$ matrix $A$ with entries in $G F(2)$ has four associated subspaces: the row space and right nullspace are orthogonal complements in $G F(2)^{n}$, and the column space and left nullspace are orthogonal complements in $G F(2)^{m}$. According to Theorem 3, we could just as easily say that an $m \times n$ matrix with entries in $G F(2)$ has four associated binary matroids, a pair of duals on an $m$-element set, and a pair of duals on an $n$-element set. For a symmetric matrix the row space and the column space are the same, and the left and right nullspace are the same.

Let $G$ be a graph. A familiar construction associates to $G$ its polygon matroid $M(G)$, the binary matroid on $E(G)$ whose circuits are the minimal edge-sets of circuits of $G$. In this paper we discuss a different way to associate a binary matroid to $G$, which was mentioned by Jaeger in 1983 [21, 22]; the notion seems to have received little attention in the intervening decades. 
Definition 4. Let $G$ be a graph, and let $\mathcal{A}(G)$ be the Boolean adjacency matrix of $G$, i.e., the $V(G) \times V(G)$ matrix with entries in $G F(2)$, in which a diagonal entry $a_{v v}$ is 1 if and only if $v$ is looped and an off-diagonal entry $a_{v w}$ is 1 if and only if $v \neq w$ are adjacent. Then the adjacency matroid $M_{A}(G)$ is the binary matroid on $V=V(G)$ represented by $\mathcal{A}(G)$, i.e., its circuits are the minimal nonempty subsets $S \subseteq V$ such that the columns of $\mathcal{A}(G)$ corresponding to elements of $S$ are linearly dependent.

Here are three comments on Definition 4:

1. We understand the term graph to include multigraphs; that is, we allow graphs to have loops and parallel edges. Although Definition 4 applies to an arbitrary graph $G, \mathcal{A}(G)$ does not reflect the number of edges connecting two adjacent vertices, or the number of loops on a looped vertex. Consequently, the reader may prefer to think of $\mathcal{A}(G)$ and $M_{A}(G)$ as defined only when $G$ is a looped simple graph.

2. In light of Theorem $3, M_{A}(G)$ may be described more simply as the binary matroid whose cycle space $Z\left(M_{A}(G)\right)$ is the nullspace of $\mathcal{A}(G)$.

3. Many graph-theoretic properties of a graph $G$ do not match conveniently with matroid-theoretic properties of $M_{A}(G)$. For example, recall that a loop in a matroid $M$ is an element $\lambda$ such that $\{\lambda\} \in \mathcal{C}(M)$, and a coloop is an element $\kappa$ such that $\kappa \notin \gamma$ for all $\gamma \in \mathcal{C}(M)$. In the polygon matroid of $G$, a loop is an edge incident on only one vertex, and a coloop is a cut edge. Propositions 5 and 6 indicate that the adjacency matroid of $G$ has very different properties: looped vertices of $G$ cannot be loops of $M_{A}(G)$, and coloops of $M_{A}(G)$ do not in general have anything to do with connectedness of $G$.

Proposition 5. A vertex $v \in V(G)$ is a loop of $M_{A}(G)$ if and only if $v$ is isolated and not looped in $G$.

Proposition 6. Suppose $v \in V(G)$, and let $G^{\prime}$ be the graph obtained from $G$ by toggling the loop status of $v$. Then $v$ is a coloop of at least one of the adjacency matroids $M_{A}(G)$, $M_{A}\left(G^{\prime}\right)$.

Proposition 5 follows immediately from Definition 4, but proving Proposition 6 requires a little more work; it follows readily from Lemma 41 below.

Theorem 3 and the equality $Z\left(M^{*}\right)=Z(M)^{\perp}$ directly imply the following.

Theorem 7. Let $G$ and $G^{\prime}$ be two n-vertex graphs, let $f: V(G) \rightarrow V\left(G^{\prime}\right)$ be a bijection, and let $2^{f}: 2^{V(G)} \rightarrow 2^{V\left(G^{\prime}\right)}$ be the isomorphism of $G F(2)$-vector spaces induced by $f$. Then the following three conditions are equivalent.

1. $f$ defines an isomorphism $M_{A}(G) \cong M_{A}\left(G^{\prime}\right)$.

2. $2^{f}$ maps the column space of $\mathcal{A}(G)$ onto the column space of $\mathcal{A}\left(G^{\prime}\right)$.

3. $2^{f}$ maps the nullspace of $\mathcal{A}(G)$ onto the nullspace of $\mathcal{A}\left(G^{\prime}\right)$.

Also, the following three conditions are equivalent.

1. $f$ defines an isomorphism $M_{A}(G) \cong M_{A}\left(G^{\prime}\right)^{*}$.

2. $2^{f}$ maps the column space of $\mathcal{A}(G)$ onto the nullspace of $\mathcal{A}\left(G^{\prime}\right)$.

3. $2^{f}$ maps the nullspace of $\mathcal{A}(G)$ onto the column space of $\mathcal{A}\left(G^{\prime}\right)$. 
Every graph with at least one edge has the same adjacency matroid as infinitely many other graphs, obtained by adjoining parallels. Even among looped simple graphs, there are many examples of nonisomorphic graphs with isomorphic adjacency matroids. For instance, the simple path of length two has the same adjacency matroid as the graph that consists of two isolated, looped vertices. However, a looped simple graph is determined up to isomorphism by the adjacency matroids of its full subgraphs.

Definition 8. Let $G$ be a graph, and suppose $S \subseteq V(G)$. Then $G[S]$ denotes the full subgraph of $G$ induced by $S$, i.e., the subgraph with $V(G[S])=S$ that includes the same incident edges as $G$.

If $v \in V(G)$ then $G[V(G) \backslash\{v\}]$ is also denoted $G-v$.

Theorem 9. Let $G$ and $G^{\prime}$ be looped simple graphs, and let $f: V(G) \rightarrow V\left(G^{\prime}\right)$ be a bijection. Then the following are equivalent.

1. $f$ is a graph isomorphism.

2. For every $S \subseteq V(G)$, $f$ defines a matroid isomorphism $M_{A}(G[S]) \cong M_{A}\left(G^{\prime}[f(S)]\right)$.

3. For every $S \subseteq V(G)$ with $|S| \leqslant 2$, the matroids $M_{A}(G[S])$ and $M_{A}\left(G^{\prime}[f(S)]\right)$ have the same nullity.

Proof. The implications $1 \Rightarrow 2 \Rightarrow 3$ are obvious. The implication $3 \Rightarrow 1$ follows from these facts: A vertex $v \in V(G)$ is looped (resp. unlooped) if and only if the nullity of $M_{A}(G[\{v\}])$ is 0 (resp. 1). If $v \neq w \in V(G)$ are both unlooped, then they are adjacent (resp. nonadjacent) in $G$ if and only if the nullity of $M_{A}(G[\{v, w\}])$ is 0 (resp. 2). If $v \in V(G)$ is looped and $w \in V(G)$ is unlooped, then they are adjacent (resp. nonadjacent) in $G$ if and only if the nullity of $M_{A}(G[\{v, w\}])$ is 0 (resp. 1). If $v \neq w \in V(G)$ are both looped, then they are adjacent (resp. nonadjacent) in $G$ if and only if the nullity of $M_{A}(G[\{v, w\}])$ is 1 (resp. 0$)$.

The polygon matroids of graphs constitute a special subclass of the binary matroids. Jaeger proved that the adjacency matroids of graphs, instead, include all the binary matroids:

Theorem 10. [21] Let $M$ be an arbitrary binary matroid. Then there is a graph $G$ with $M=M_{A}(G)$.

Jaeger also gave an original characterization of the polygon matroids of graphs, which involves interlacement in 4-regular graphs. Recall that a connected 4-regular graph has at least one Euler circuit, i.e., a closed trail that includes every edge. In general, a 4-regular graph has at least one Euler system, which includes one Euler circuit of each connected component. An Euler system $C$ of a 4-regular graph $F$ has an associated alternance graph or interlacement graph $\mathcal{I}(C)$, a simple graph defined as follows [7, 27]: The vertex-set of $\mathcal{I}(C)$ is $V(F)$, and two vertices $v \neq w$ are adjacent in $\mathcal{I}(C)$ if and only if there is some circuit of $C$ on which they appear in the order $v \ldots w \ldots v \ldots w$.

Theorem 11. [22] The polygon matroids of graphs are the duals of the adjacency matroids of looped interlacement graphs. 
We recall the proofs of Theorems 3 and 10 in Section 2, and in Sections 3 and 4 we give a new proof of Theorem 11. This new proof consists of two parts, which are interesting enough to state separately. (See Section 3 for definitions.)

Theorem 12. If $P$ is a circuit partition of a 4-regular graph $F$ and $C$ is an Euler system of $F$ that is compatible with $P$, then the polygon matroid of the touch-graph of $P$ is dual to the adjacency matroid of a particular looped version of the interlacement graph of $C$.

Theorem 13. Every graph without isolated, unlooped vertices is the touch-graph of some circuit partition in some 4-regular graph.

Theorems 12 and 13 are not of only abstract interest. Touch-graphs of circuit partitions in 4-regular graphs are fairly easy to understand, and many properties of general adjacency matroids may be motivated by dualizing properties of touch-graphs. It turns out that the general theory of adjacency matroids is closely connected to two important notions that also generalize properties of 4-regular graphs: the $\Delta$-matroids introduced by Bouchet $[9,10,11]$ and the interlace polynomials introduced by Arratia, Bollobás and Sorkin $[2,3,4]$. These close connections are indicated by the fact that local complementation plays a significant role in all three theories.

Definition 14. Let $G$ be a graph with a vertex $v$. Then the local complement $G^{v}$ is the looped simple graph obtained from $G$ by toggling the loop status of every neighbor of $v$, and toggling the adjacency status of every pair of neighbors of $v$.

In this definition (and throughout the paper) we use the term neighbors to describe distinct, adjacent vertices. Consequently, local complementation may be described more explicitly as follows: $G^{v}$ is the looped simple graph related to $G$ as follows: if $v \neq w \neq$ $x \neq v$ and both $w$ and $x$ are neighbors of $v$ in $G$, then $G^{v}$ has an edge $w x$ if and only if $w$ and $x$ are not adjacent in $G$; if $w \neq x \in V(G)$ and at least one of $w, x$ is not a neighbor of $v$ in $G$, then $G^{v}$ has an edge $w x$ if and only if $w$ and $x$ are adjacent in $G$; if $w \neq v$ is a neighbor of $v$ in $G$ then there is a loop on $w$ in $G^{v}$ if and only if $w$ is not looped in $G$; and if $w$ is not a neighbor of $v$ in $G$ then there is a loop on $w$ in $G^{v}$ if and only if $w$ is looped in $G$. Note that for every graph $G,\left(G^{v}\right)^{v}$ is the looped simple graph obtained from $G$ by replacing each set of parallels with a single edge.

There are two matroid minor operations, deletion and contraction.

Definition 15. If $M$ is a matroid on $V$ and $v \in V$ then the deletion $M-v$ is the matroid on $V \backslash\{v\}$ with $\mathcal{C}(M-v)=\{\gamma \in \mathcal{C}(M) \mid v \notin \gamma\}$.

If $M$ is a binary matroid, then $M-v$ is the binary matroid on $V \backslash\{v\}$ with $Z(M-v)=$ $Z(M) \cap 2^{V \backslash\{v\}}$.

Definition 16. If $M$ is a matroid on $V$ and $v \in V$, then the contraction $M / v$ is the matroid on $V \backslash\{v\}$ with $\mathcal{C}(M / v)=\{$ minimal nonempty subsets $\gamma \subseteq V \backslash\{v\} \mid \gamma \cup\{v\}$ contains an element of $\mathcal{C}(M)\}$. 
If $M$ is a binary matroid, let $[v]$ denote the subspace of $2^{V}$ spanned by $\{v\}$; we identify $2^{V \backslash\{v\}}$ with $2^{V} /[v]$ in the natural way. Then $M / v$ is the binary matroid on $V \backslash\{v\}$ with $Z(M / v)=(Z(M)+[v]) /[v]$.

Our first indication of the importance of local complementation for adjacency matroids is the fact that the matroid minors $M_{A}(G) / v$ and $M_{A}(G)-v$ can always be obtained by deleting $v$ from graphs related to $G$ through local complementation.

Theorem 17. If $v \in V(G)$ is a looped vertex then $M_{A}(G) / v=M_{A}\left(G^{v}-v\right)$.

Theorem 18. Suppose $v$ is an unlooped vertex of $G$.

1. If $v$ is isolated then $M_{A}(G) / v=M_{A}(G-v)$.

2. If $w$ is an unlooped neighbor of $v$ then $M_{A}(G) / v=M_{A}\left(\left(G^{w}\right)^{v}-v\right)$.

3. If $w$ is a looped neighbor of $G$ then $M_{A}(G) / v=M_{A}\left(\left(\left(G^{v}\right)^{w}\right)^{v}-v\right)$.

Theorem 19. If $v$ is not a coloop of $M_{A}(G)$ then $M_{A}(G)-v=M_{A}(G-v)$.

In general, Theorem 19 fails for coloops. For example, let $v$ and $w$ be the vertices of the simple path $P_{2}$ of length two. Then $w$ is isolated and unlooped in $P_{2}-v$; consequently $\mathcal{C}\left(M_{A}\left(P_{2}-v\right)\right)=\{\{w\}\}$ even though $\mathcal{C}\left(M_{A}\left(P_{2}\right)\right)=\varnothing$.

Observe that if $M$ is a matroid on $V$ and $v \in V$, then $M-v=M / v$ if and only if $v$ is either a loop or a coloop. (For if $v$ appears in any circuit $\gamma$ with $|\gamma|>1$ then $\gamma \backslash\{v\}$ contains a circuit of $M / v$ but $\gamma \backslash\{v\}$ contains no circuit of $M-v$.) It follows that the failure of Theorem 19 for coloops is not a significant inconvenience: if $v$ is a coloop of $M_{A}(G)$ then we may refer to Theorem 17 or Theorem 18 to describe $M_{A}(G)-v=$ $M_{A}(G) / v$.

Another instance of the importance of local complementation is the fact that matroid deletions from $M_{A}(G)$ and $M_{A}\left(G^{v}\right)$ always coincide.

Theorem 20. If $v \in V(G)$ then $M_{A}(G)-v=M_{A}\left(G^{v}\right)-v$.

The connection between the entire matroids $M_{A}(G)$ and $M_{A}\left(G^{v}\right)$ is more complicated. Recall that if $M$ and $M^{\prime}$ are matroids on disjoint sets $V$ and $V^{\prime}$ then their direct sum $M \oplus M^{\prime}$ is the matroid on $V \cup V^{\prime}$ with $\mathcal{C}\left(M \oplus M^{\prime}\right)=\mathcal{C}(M) \cup \mathcal{C}\left(M^{\prime}\right)$. Also, if $v$ is a single element then $U_{1,1}(\{v\})$ denotes the matroid on $\{v\}$ in which $v$ is a coloop, and $U_{1,0}(\{v\})$ denotes the matroid on $\{v\}$ in which $v$ is a loop. Clearly $M=(M-v) \oplus U_{1,1}(\{v\})$ if and only if $v$ is a coloop of $M$, and $M=(M-v) \oplus U_{1,0}(\{v\})$ if and only if $v$ is a loop of $M$.

Theorem 21. 1. If $v \in V(G)$ is unlooped then $M_{A}\left(G^{v}\right)=M_{A}(G)$.

2. If $v \in V(G)$ is a coloop of both $M_{A}(G)$ and $M_{A}\left(G^{v}\right)$, then $M_{A}\left(G^{v}\right)=M_{A}(G)$.

3. If $v \in V(G)$ is looped and not a coloop of one of $M_{A}(G), M_{A}\left(G^{v}\right)$, then $v$ is a coloop of the other and $M_{A}\left(G^{v}\right) \nsucceq M_{A}(G)$. More specifically, if $\left\{M_{A}(G), M_{A}\left(G^{v}\right)\right\}=\left\{M_{1}, M_{2}\right\}$ with $v$ not a coloop of $M_{1}$, then $v$ is a coloop of $M_{2}$ and $M_{2}=\left(M_{1}-v\right) \oplus U_{1,1}(\{v\})$. 
Unlike Theorem 7, Theorem 21 does not explain all isomorphisms of adjacency matroids. For instance, the simple path of length two has the same adjacency matroid as a disconnected graph consisting of two looped vertices; but local complementation cannot disconnect a connected graph.

Theorems 17 - 21 are proven in Section 5, using elementary linear algebra. In Section 6 we discuss Theorem 23, which provides another illustration of the connections tying adjacency matroids to delta-matroids and the theory of the interlace polynomials. It is a matroid version of Lemma 2 of Balister, Bollobás, Cutler, and Pebody [5]. A preliminary definition will be useful.

Definition 22. If $v$ is a vertex of $G$ then $G(v)$ denotes the graph obtained from $G$ by removing every loop incident on $v, G(v, \ell)$ denotes the graph obtained from $G(v)$ by attaching a loop at $v$, and $G(v, \ell i)$ denotes the graph obtained from $G(v, \ell)$ by isolating $v$ (i.e., removing all non-loop edges incident on $v$ ).

Theorem 23. Let $v$ be a vertex of $G$. Then two of the three adjacency matroids $M_{A}(G(v))$, $M_{A}(G(v, \ell)), M_{A}(G(v, \ell i))$ are the same, and the other is different. The cycle space of the different matroid contains the cycle space shared by the two that are the same, and its dimension is greater by 1.

The adjacency matroid $M_{A}(G(v, \ell i))$ may be described in two other ways. As $v$ is an isolated, looped vertex of $G(v, \ell i)$, it is a coloop of $M_{A}(G(v, \ell i))$; hence $M_{A}(G(v, \ell i))=$ $M_{A}(G(v, \ell i)-v) \oplus U_{1,1}(\{v\})=M_{A}(G-v) \oplus U_{1,1}(\{v\})$. Another description comes from Theorem 17, which tells us that $M_{A}(G-v)=M_{A}\left(G^{v}(v, \ell)\right) / v$. Also, the fact that $v$ is not a loop of $M_{A}\left(G^{v}(v, \ell)\right)$ implies that $M_{A}\left(G^{v}(v, \ell)\right) / v$ and $M_{A}\left(G^{v}(v, \ell)\right)$ have the same nullity. For ease of reference we state these observations as a proposition.

Proposition 24. If $v \in V(G)$ then $v$ is a coloop of

$$
M_{A}(G(v, \ell i))=M_{A}(G-v) \oplus U_{1,1}(\{v\})=\left(M_{A}\left(G^{v}(v, \ell)\right) / v\right) \oplus U_{1,1}(\{v\}) .
$$

Consequently $M_{A}(G(v, \ell i)), M_{A}(G-v), M_{A}\left(G^{v}(v, \ell)\right) / v$ and $M_{A}\left(G^{v}(v, \ell)\right)$ all have the same nullity.

Recall that according to Proposition 6, $v$ must be a coloop of at least one of the matroids $M_{A}(G(v)), M_{A}(G(v, \ell))$. Consequently $v$ must fall under one (and only one) of these cases:

1. $v$ is a coloop of both $M_{A}(G(v))$ and $M_{A}(G(v, \ell))$

2. $v$ is a coloop of $M_{A}(G(v))$ and not $M_{A}(G(v, \ell))$

3. $v$ is a coloop of $M_{A}(G(v, \ell))$ and not $M_{A}(G(v))$

As each vertex of $G$ must fall under precisely one of the cases $1-3$, we obtain a partition of $V(G)$ into three subsets. We refer to this partition of $V(G)$ as the principal vertex tripartition of $G$. In Section 7 we prove that the three subsets of the principal 
vertex tripartition correspond precisely to the three alternatives of Theorem 23: $v$ falls under case 1 if and only if $M_{A}(G(v))=M_{A}(G(v, \ell))$; $v$ falls under case 2 if and only if $M_{A}(G(v))=M_{A}(G(v, \ell i))$; and $v$ falls under case 3 if and only if $M_{A}(G(v, \ell))=$ $M_{A}(G(v, \ell i))$. Moreover, $v$ falls under case 1 in $G$ if and only if $v$ falls under case 2 in $G^{v}$, and vice versa. Theorem 25 below includes all of these results, and also gives a few more details; in particular, the final assertion of case 1 corrects an error in [29].

Theorem 25. Let $v$ be a vertex of $G$. Then the list $M_{A}(G(v)), M_{A}(G(v, \ell)), M_{A}(G(v, \ell i))$, $M_{A}\left(G^{v}(v)\right), M_{A}\left(G^{v}(v, \ell)\right), M_{A}\left(G^{v}(v, \ell i)\right)$ includes either two or three distinct matroids. Only one of these distinct matroids does not include $v$ as a coloop, and this matroid determines the others as follows.

1. If $v$ is a coloop of both $M_{A}(G(v))$ and $M_{A}(G(v, \ell))$ then $v$ is not a coloop of $M_{A}\left(G^{v}(v, \ell)\right)$,

$$
\begin{aligned}
M_{A}(G(v, \ell i)) & =\left(M_{A}\left(G^{v}(v, \ell)\right) / v\right) \oplus U_{1,1}(\{v\}) \text { and } \\
M_{A}(G(v)) & =M_{A}(G(v, \ell))=M_{A}\left(G^{v}(v)\right)=M_{A}\left(G^{v}(v, \ell i)\right) \\
& =\left(M_{A}\left(G^{v}(v, \ell)\right)-v\right) \oplus U_{1,1}(\{v\}) .
\end{aligned}
$$

Moreover, $M_{A}(G(v, \ell i))$ and $M_{A}\left(G^{v}(v, \ell)\right)$ have the same nullity, say $\nu+1$; the nullity of $M_{A}(G(v))$ is $\nu$ and

$$
Z\left(M_{A}(G(v, \ell i))\right) \cap Z\left(M_{A}\left(G^{v}(v, \ell)\right)\right)=Z\left(M_{A}(G(v))\right) .
$$

This case requires that $G^{v}-v$ have at least one looped vertex.

2. If $v$ is not a coloop of $M_{A}(G(v, \ell))$ then the assertions of case 1 hold, with the roles of $G$ and $G^{v}$ interchanged.

3. If $v$ is not a coloop of $M_{A}(G(v))$ then

$$
\begin{aligned}
M_{A}\left(G^{v}(v)\right) & =M_{A}(G(v)) \text { and } \\
M_{A}(G(v, \ell)) & =M_{A}\left(G^{v}(v, \ell)\right)=M_{A}(G(v, \ell i))=M_{A}\left(G^{v}(v, \ell i)\right) \\
& =\left(M_{A}(G(v))-v\right) \oplus U_{1,1}(\{v\}) .
\end{aligned}
$$

Moreover, the nullity of $M_{A}(G(v))$ is 1 more than the nullity of $M_{A}(G(v, \ell))$, and

$$
Z\left(M_{A}(G(v, \ell))\right) \subset Z\left(M_{A}(G(v))\right) .
$$

The principal vertex tripartition is reminiscent of the principal edge tripartition of Rosenstiehl and Read [28], but there is a fundamental difference between the two tripartitions. The principal edge tripartition of $G$ is determined by the polygon matroid of $G$, but the principal vertex tripartition of $G$ is not determined by the adjacency matroid of $G$ :

Theorem 26. The adjacency matroid and the principal vertex tripartition are independent, in the sense that two graphs may have isomorphic adjacency matroids and distinct principal vertex tripartitions, or nonisomorphic adjacency matroids and equivalent principal vertex tripartitions. 
After verifying Theorem 26 in Section 8, in Sections 9 - 11 we turn our attention to the close connection between adjacency matroids and $\Delta$-matroids.

Definition 27. [9] $A$ delta-matroid ( $\Delta$-matroid for short) is an ordered pair $D=(V, \sigma)$ consisting of a finite set $V$ and a nonempty family $\sigma \subseteq 2^{V}$ that satisfies the symmetric exchange axiom: For all $X, Y \in \sigma$ and all $u \in X \Delta Y, X \Delta\{u\} \in \sigma$ or there is a $v \in X \Delta Y$ such that $v \neq u$ and $X \Delta\{u, v\} \in \sigma$ (or both).

We often write $X \in D$ rather than $X \in \sigma$. The name $\Delta$-matroid reflects the fact that if $M$ is a matroid, the family of bases $\mathcal{B}(M)$ satisfies the symmetric exchange property; indeed $\mathcal{B}(M)$ satisfies the stronger basis exchange axiom: if $X, Y \in \mathcal{B}(M)$ and $u \in X \backslash Y$ then there is some $v \in Y \backslash X$ with $X \Delta\{u, v\} \in \mathcal{B}(M)$.

Definition 28. [9] If $G$ is a graph then its associated $\Delta$-matroid is $\mathcal{D}_{G}=(V(G), \sigma)$ with

$$
\sigma=\{S \subseteq V(G) \mid \mathcal{A}(G[S]) \text { is nonsingular }\} .
$$

$\mathcal{D}_{G}$ is determined by the adjacency matroids $M_{A}(G[S]): S \in \mathcal{D}_{G}$ if and only if $M_{A}(G[S])$ is a free matroid (i.e., $\mathcal{C}\left(M_{A}(G[S])\right)=\varnothing$ ). There is more to the relationship between $\mathcal{D}_{G}$ and the matroids $M_{A}(G[S])$ than this obvious observation, though. Recall that if $M$ is a matroid on $V$ then an independent set of $M$ is a subset of $V$ that contains no circuit of $M$, and a basis of $M$ is a maximal independent set. For $S \subseteq V(G)$ let $\mathcal{I}\left(M_{A}(G[S])\right)$ and $\mathcal{B}\left(M_{A}(G[S])\right)$ denote the families of independent sets and bases (respectively) of the adjacency matroid $M_{A}(G[S])$.

Theorem 29. Let $G$ be a graph, and suppose $S \subseteq V(G)$.

1. $M_{A}(G[S])$ is the matroid on $S$ with

$$
\mathcal{B}\left(M_{A}(G[S])\right)=\left\{\text { maximal } B \subseteq S \mid B \in \mathcal{D}_{G}\right\}
$$

2. $M_{A}(G[S])$ is the matroid on $S$ with

$$
\mathcal{I}\left(M_{A}(G[S])\right)=\left\{I \subseteq S \mid \text { there is some } X \in \mathcal{D}_{G} \text { with } I \subseteq X \subseteq S\right\} .
$$

3. $\mathcal{D}_{G[S]}$ is the $\Delta$-matroid $(S, \sigma)$ with

$$
\sigma=\bigcup_{T \subseteq S} \mathcal{B}\left(M_{A}(G[T])\right) .
$$

Although Theorem 29 applies to arbitrary subsets $S \subseteq V(G)$, the heart of the theorem is the result that part 1 holds for $S=V(G)$; as noted by Brijder and Hoogeboom [14], this result is a special case of the strong principal minor theorem of Kodiyalam, Lam and Swan [24].

In Sections 10 and 11 we reprove Theorems $17-21$ within the contexts of set systems and $\Delta$-matroids. In particular, Theorem 17 and Theorem 19 are generalized to set systems and $\Delta$-matroids, respectively. In a similar vein, Theorem 5.6 of [14] shows that some 
aspects of the principal vertex tripartition extend to matroids associated with arbitrary $\Delta$-matroids.

In Section 12 we discuss the connection between the interlace polynomials introduced by Arratia, Bollobás and Sorkin $[2,3,4]$ and the Tutte polynomials of the adjacency matroids of a graph and its full subgraphs. This connection seems to be fundamentally different from the connection between the one-variable interlace polynomial of a planar circle graph and the Tutte polynomial of an associated checkerboard graph, discussed by Arratia, Bollobás and Sorkin [3] and Ellis-Monaghan and Sarmiento [18].

\section{Theorems 3 and 10}

For the convenience of the reader, in this section we provide proofs of theorems of GhouilaHouri [19] and Jaeger [21] mentioned in the introduction.

\subsection{Proof of Theorem 3}

It is well known that axiom 4 of Definition 1 may be replaced by the following seemingly stronger requirement $[26,35,37]$ :

$4^{\prime}$. If $C_{1}, C_{2}, \ldots, C_{k} \in \mathcal{C}(M)$ do not sum to $\varnothing$ in $2^{V}$ then there are pairwise disjoint $C_{1}^{\prime}, \ldots, C_{k^{\prime}}^{\prime} \in \mathcal{C}(M)$ such that

$$
\sum_{i=1}^{k} C_{i}=\bigcup_{i=1}^{k^{\prime}} C_{i}^{\prime}
$$

This axiom is useful in the proof of Theorem 3:

Let $M$ be a binary matroid on $V$. We can certainly construct $Z(M)$ from $\mathcal{C}(M)$, using the addition of $2^{V}$. It turns out that we can also construct $\mathcal{C}(M)$ from $Z(M)$ :

$$
\mathcal{C}(M)=\{\text { minimal nonempty subsets of } V \text { that appear in } Z(M)\} .
$$

The proof is simple: axiom $4^{\prime}$ implies that every nonempty element of $Z(M)$ contains a circuit, so every minimal nonempty element of $Z(M)$ is an element of $\mathcal{C}(M)$; conversely, axiom 3 tells us that no circuit contains another, so it is impossible for a circuit to contain a minimal nonempty element of $Z(M)$ other than itself.

This implies that the function $Z$ is injective.

Now, let $W$ be any subspace of $2^{V}$. If $W=\{\varnothing\}$ then $W=Z(U)$, where $U$ is the free matroid on $V$ (i.e., $\mathcal{C}(U)=\varnothing$ ). Suppose $\operatorname{dim} W \geqslant 1$, and let $\mathcal{C}(W)$ be the set of minimal nonempty subsets of $V$ that appear in $W$. It is a simple matter to verify that $\mathcal{C}(W)$ satisfies Definition 1; hence $\mathcal{C}(W)$ is the circuit-set of a binary matroid $M(W)$. As $\mathcal{C}(M(W))=\mathcal{C}(W) \subseteq W$, and $Z(M(W))$ is spanned by $\mathcal{C}(M(W)), Z(M(W))$ is a subspace of $W$.

Could $Z(M(W))$ be a proper subspace of $W$ ? If so, then there is some $w \in W$ that is not an element of $Z(M(W))$. By definition, $\mathcal{C}(W)$ must include some element $\gamma$ that is a subset of $w$. Then $w+\gamma=w \Delta \gamma=w \backslash \gamma$ is also an element of $W-Z(M(W))$, and its 
cardinality is strictly less than that of $w$. We deduce that $W-Z(M(W))$ does not have an element of smallest cardinality. This is ridiculous, so $Z(M(W))$ cannot be a proper subspace of $W$.

It follows that the function $Z$ is surjective.

\subsection{Proof of Theorem 10}

In light of Theorems 3 and 7 of Section 1, proving Theorem 10 is the same as proving the following.

Proposition 30. Let $A$ be a $k \times n$ matrix with entries in $G F(2)$. Then there is a symmetric $n \times n$ matrix $B$ whose nullspace is the same as the right nullspace of $A$.

Proof. If the right nullspace space of $A$ is $G F(2)^{n}$, the proposition is satisfied by the zero matrix; if the right nullspace of $A$ is $\{\mathbf{0}\}$ then the proposition is satisfied by the identity matrix.

Otherwise, the right nullspace of $A$ is a proper subspace of $G F(2)^{n}$. Using elementary row operations, we obtain from $A$ an $r \times n$ matrix $C$ in echelon form, which has the same right nullspace as $A$. (Here $r$ is the rank of $A$.) There is a permutation $\pi$ of $\{1, \ldots, n\}$ such that the matrix obtained by permuting the columns of $C$ according to $\pi$ is of the form

$$
C^{\prime}=\left(\begin{array}{ll}
I_{r} & C^{\prime \prime}
\end{array}\right)
$$

where $I_{r}$ is an identity matrix. If $\left(C^{\prime \prime}\right)^{\operatorname{tr}}$ denotes the transpose of $C^{\prime \prime}$, then

$$
B^{\prime}=\left(\begin{array}{cc}
I_{r} & C^{\prime \prime} \\
\left(C^{\prime \prime}\right)^{t r} & \left(C^{\prime \prime}\right)^{t r} \cdot C^{\prime \prime}
\end{array}\right)
$$

is a symmetric matrix. $B^{\prime}$ has the same right nullspace as $C^{\prime}$, for if $\left(\begin{array}{ll}I_{r} & C^{\prime \prime}\end{array}\right) \cdot \kappa=\mathbf{0}$ then certainly

$$
\left(\left(C^{\prime \prime}\right)^{t r} \quad\left(C^{\prime \prime}\right)^{t r} \cdot C^{\prime \prime}\right) \cdot \kappa=\left(C^{\prime \prime}\right)^{t r} \cdot\left(\begin{array}{ll}
I_{r} & C^{\prime \prime}
\end{array}\right) \cdot \kappa=\mathbf{0} .
$$

Consequently, a matrix $B$ satisfying the statement may be obtained by permuting the rows and columns of $B^{\prime}$ according to $\pi^{-1}$.

\section{Theorem 12}

Our proof of Theorem 11 is rather different from Jaeger's original argument [22]. We begin with the definition of touch-graphs, which appeared implicitly in Jaeger's later work [23] and were subsequently discussed explicitly by Bouchet [8]. Recall that a trail in a graph is a walk which may include repeated vertices, but may not include repeated edges. A closed trail is also called a circuit; one such circuit may contain another, so it is important to distinguish these circuits from matroid circuits.

Definition 31. Let $F$ be a 4-regular graph. A circuit partition or Eulerian decomposition of $F$ is a partition $P$ of the edge-set $E(F)$ into pairwise disjoint subsets, each of which is the edge-set of a closed trail in $F$. 
Definition 32. If $P$ is a circuit partition of $F$ then the touch-graph $T c h(P)$ is a graph with a vertex for each element of $P$ and an edge for each vertex of $F$; the edge corresponding to $v \in V(F)$ is incident on the vertex or vertices corresponding to element(s) of $P$ incident at $v$.

Observe that a walk $v_{1}, e_{1}, v_{2}, \ldots, e_{k-1}, v_{k}$ in $F$ gives rise to a walk in $T \operatorname{ch}(P)$; for $1<i<k$ the edge of $T \operatorname{ch}(P)$ corresponding to $v_{i}$ connects the vertex or vertices of $T c h(P)$ corresponding to the circuit(s) of $P$ containing $e_{i-1}$ and $e_{i}$. Also, adjacent vertices of $T \operatorname{ch}(P)$ must correspond to circuits of $P$ contained in a single connected component of $F$, because the edge of $T \operatorname{ch}(P)$ connecting them corresponds to a vertex of $F$ incident on both circuits. Consequently, there is a natural correspondence between the connected components of $F$ and those of $T \operatorname{ch}(P)$.

As mentioned in the introduction, an Euler system of a 4-regular graph $F$ is a set that includes one Euler circuit of each connected component of $F$. The alternance graph or interlacement graph of an Euler system $C$ of $F$ is the simple graph $\mathcal{I}(C)$ with $V(\mathcal{I}(C))=$ $V(F)$, in which two vertices $v \neq w$ are adjacent if and only if they appear in the order $v \ldots w \ldots v \ldots w$ on some circuit of $C[7,27]$. We also use $\mathcal{I}(C)$ to denote the adjacency matrix $\mathcal{A}(\mathcal{I}(C))$.

Suppose $P$ is an arbitrary circuit partition of $F, C$ is an arbitrary Euler system of $F$, and $v \in V(F)$. Choose a preferred orientation of each circuit of $C$, and use these orientations to direct the edges of $F$. Suppose we choose a circuit of $P$ that is incident on $v$, and we walk toward $v$ on an edge $e$ of this circuit that is in-directed according to the preferred orientation. If we continue to follow this circuit of $P$, how do we leave $v$ ? There are three possibilities: $(\phi)$ we leave on the out-directed edge we would use if we were following the incident circuit of $C,(\chi)$ we leave on the out-directed edge we would not use if we were following the incident circuit of $C$, or $(\psi)$ we leave on the in-directed edge we did not use before.

It is not difficult to see that the $\phi, \chi, \psi$ designation at $v$ would be the same if we were to choose the other in-directed edge instead of $e$, or if we were to choose the other orientation of the incident circuit of $C$. Consequently we run no risk of confusion by referring to the $\phi, \chi, \psi$ designation as the transition of $P$ with respect to $C$ at $v$. Clearly $P$ is determined by its transitions with respect to any Euler system, and each of the $3^{|V(F)|}$ systems of choices of transitions yields a circuit partition of $F$.

Definition 33. Under these circumstances, the relative interlacement graph $\mathcal{I}_{P}(C)$ is obtained from $\mathcal{I}(C)$ by removing each vertex of type $\phi$, and attaching a loop to each vertex of type $\psi$.

We also use $\mathcal{I}_{P}(C)$ to denote the adjacency matrix $\mathcal{A}\left(\mathcal{I}_{P}(C)\right)$. An important property of $\mathcal{I}_{P}(C)$ is the circuit-nullity formula:

Theorem 34. If $F$ has $c(F)$ connected components then

$$
\nu\left(\mathcal{I}_{P}(C)\right)=|P|-c(F),
$$

where $\nu$ denotes the GF(2)-nullity. 


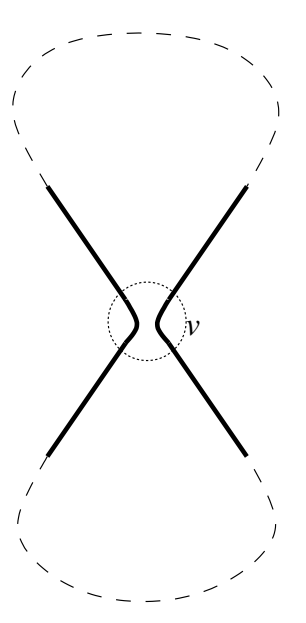

(a)

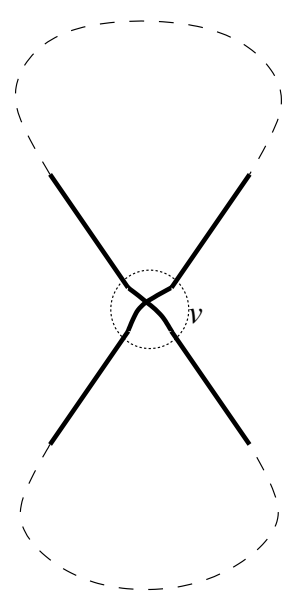

(b)

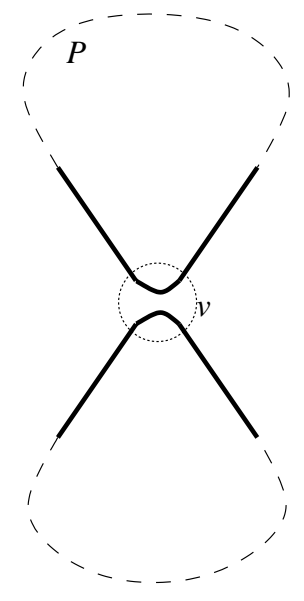

(c)

Figure 1: Given choices of transitions at other vertices, two of the three transitions at $v$ produce touch-graphs in which $v$ is a loop.

We refer to $[30,31,33]$ for discussions and proofs of the circuit-nullity formula. These references are recent, but special cases of Theorem 34 have been known for almost 100 years; the earliest of these special cases seems to be the one that appears in Brahana's study of systems of curves on surfaces [12]. Jaeger [23] proved the special case of the circuit-nullity formula in which $C$ and $P$ are compatible, i.e., $P$ does not involve the $\phi$ transition with respect to $C$ at any vertex of $F$. This special case is more general than it might appear at first glance, as Kotzig [25] proved that if $P$ is any circuit partition of $F$, then $F$ has an Euler system $C$ that is compatible with $P$.

Observe that the rank of the polygon matroid of $T \operatorname{ch}(P)$ is $|V(T \operatorname{ch}(P))|-c(T \operatorname{ch}(P))$, which equals $|P|-c(F)$. The circuit-nullity formula tells us that this rank equals $\nu\left(\mathcal{I}_{P}(C)\right.$ ). In case $C$ and $P$ are compatible, Jaeger sharpened this equality by identifying the nullspace of $\mathcal{I}_{P}(C)$ :

Theorem 35. [23] Let $F$ be a 4-regular graph with a circuit partition $P$ and a compatible Euler system $C$. Then the nullspace of $\mathcal{I}_{P}(C)$ and the cycle space of $T c h(P)$ are orthogonal complements in $G F(2)^{V(F)}$.

Theorem 35 suffices for our present purposes, but we might mention that a modified version of the result holds in the general (non-compatible) case; see [33].

In view of Theorem 3, Theorem 35 is equivalent to the following precise version of Theorem 12:

Theorem 36. Let $F$ be a 4-regular graph with a circuit partition $P$ and a compatible Euler system $C$. Then $M_{A}\left(\mathcal{I}_{P}(C)\right)^{*}$ is the polygon matroid of $T c h(P)$.

We sketch a quick proof of Theorem 36, which uses the circuit-nullity formula. 
Lemma 37. Suppose $F$ is a 4-regular graph with a circuit partition $P$ and an Euler system $C$, and suppose $x$ is a vertex of $\mathcal{I}_{P}(C)$. Two different circuits of $P$ are incident at $x$ if and only if $\rho\left(\mathcal{I}_{P}(C)\right)=\rho\left(\mathcal{I}_{P}(C)-x\right)$.

Proof. Let $P^{\prime}$ be the circuit partition obtained from $P$ by changing the transition at $x$ : the circuit of $P^{\prime}$ incident at $x$ follows the circuit of $C$ incident at $x$.

If two different circuits of $P$ are incident at $x$, then $P^{\prime}$ is obtained from $P$ by uniting these two circuits. (See Figure 1.) It follows from the circuit-nullity formula that $\nu\left(\mathcal{I}_{P^{\prime}}(C)\right)=\nu\left(\mathcal{I}_{P}(C)\right)-1$. As $\mathcal{I}_{P^{\prime}}(C)$ is $\mathcal{I}_{P}(C)-x$, we conclude that $\rho\left(\mathcal{I}_{P}(C)\right)=$ $\rho\left(\mathcal{I}_{P}(C)-x\right)$. Conversely, if $\rho\left(\mathcal{I}_{P}(C)\right)=\rho\left(\mathcal{I}_{P}(C)-x\right)$ then $\nu\left(\mathcal{I}_{P}(C)\right)=1+\nu\left(\mathcal{I}_{P^{\prime}}(C)\right)$, so the circuit-nullity formula tells us that $|P|=1+\left|P^{\prime}\right|$; consequently two different circuits of $P$ are incident at $x$.

Recall that an independent set of a matroid is a set that contains no circuit.

Corollary 38. Let $F$ be a 4-regular graph with a circuit partition $P$ and an Euler system $C$. Suppose $k \geqslant 1$ and $X=\left\{x_{1}, \ldots, x_{k}\right\} \subseteq V\left(\mathcal{I}_{P}(C)\right)$. For $1 \leqslant i \leqslant k$ let $P_{i}$ be the circuit partition that involves the same transition as $C$ at each of $x_{1}, \ldots, x_{i}$, and the same transition as $P$ at every other vertex. Then the following are equivalent:

1. $X$ is an independent set of the polygon matroid of Tch $(P)$.

2. $\rho\left(\mathcal{I}_{P}(C)-X\right)=\rho\left(\mathcal{I}_{P}(C)\right)$.

3. $\left|P_{i}\right|=|P|-i$ for each $i \geqslant 1$.

Proof. If $X=\varnothing$ then all three properties hold, and if $|X|=1$ then their equivalence follows from Lemma 37 and the fact that $x_{1}$ is a non-loop edge of $T c h(P)$ if and only if two different circuits of $P$ are incident there.

We proceed by induction on $|X|=k>1$. Observe that $\mathcal{I}_{P_{i}}(C)=\mathcal{I}_{P}(C)-\left\{x_{1}, \ldots, x_{i}\right\}$ for each $i>0$.

If any one of the three properties fails for $\left\{x_{1}, \ldots, x_{k-1}\right\}$ then by induction, all three fail for $\left\{x_{1}, \ldots, x_{k-1}\right\}$; clearly then all three also fail for $X$.

Suppose all three properties hold for $\left\{x_{1}, \ldots, x_{k-1}\right\}$. Property 3 implies that if $1 \leqslant i<$ $k$, then $T c h\left(P_{i}\right)$ is obtained from $T c h(P)$ by first contracting the edges corresponding to $x_{1}, \ldots, x_{i}$, and then attaching loops to the vertices of $T \operatorname{ch}(P) /\left\{x_{1}, \ldots, x_{i}\right\}$ corresponding to $x_{1}, \ldots, x_{i}$.

If property 1 holds for $X$ then $x_{k}$ is a non-loop edge in $T \operatorname{ch}(P) /\left\{x_{1}, \ldots, x_{k-1}\right\}$, and hence also in $T c h\left(P_{k-1}\right)$. That is, two different circuits of $P_{k-1}$ are incident at $x_{k}$. Lemma 37 then implies that $\rho\left(\mathcal{I}_{P_{k-1}}(C)-x_{k}\right)=\rho\left(\mathcal{I}_{P_{k-1}}(C)\right)$; this equals $\rho\left(\mathcal{I}_{P}(C)\right)$ because property 2 holds for $\left\{x_{1}, \ldots, x_{k-1}\right\}$. As $\mathcal{I}_{P}(C)-X=\mathcal{I}_{P_{k-1}}(C)-x_{k}$, property 2 holds for $X$.

If property 2 holds for $X$ then as property 2 also holds for $\left\{x_{1}, \ldots, x_{k-1}\right\}, \rho\left(\mathcal{I}_{P_{k-1}}(C)\right)$ $=\rho\left(\mathcal{I}_{P_{k-1}}(C)-x_{k}\right)$. Lemma 37 tells us that two different circuits of $P_{k-1}$ are incident at $x_{k}$, so $\left|P_{k}\right|=\left|P_{k-1}\right|-1$. As property 3 holds for $\left\{x_{1}, \ldots, x_{k-1}\right\}$, it also holds for $X$.

Finally, if property 3 holds for $X$ then $\left|P_{k}\right|=\left|P_{k-1}\right|-1$, so two different circuits of $P_{k-1}$ are incident at $x_{k}$. That is, the edge of $T \operatorname{ch}\left(P_{k-1}\right)$ corresponding to $x_{k}$ is not a loop. It 
follows that the edge of $T \operatorname{ch}(P)$ corresponding to $x_{k}$ is not a loop in $T \operatorname{ch}(P) /\left\{x_{1}, \ldots, x_{k-1}\right\}$. As property 1 holds for $\left\{x_{1}, \ldots, x_{k-1}\right\}$, it follows that $X$ is independent in $T \operatorname{ch}(P)$.

Note by the way that property 1 of Corollary 38 is independent of the choice of an order for the elements of $X$, so despite its appearance, property 3 must also be independent of order.

The last step of the proof of Theorem 36 is the following.

Proposition 39. Let $F$ be a 4-regular graph with a circuit partition $P$ and an Euler system $C$. Suppose $X=\left\{x_{1}, \ldots, x_{k}\right\} \subseteq V\left(\mathcal{I}_{P}(C)\right)$. Then $\rho\left(\mathcal{I}_{P}(C)-X\right)=\rho\left(\mathcal{I}_{P}(C)\right)$ if and only if $X$ is an independent set of $M_{A}\left(\mathcal{I}_{P}(C)\right)^{*}$.

Proof. Recall that $M_{A}\left(\mathcal{I}_{P}(C)\right)^{*}$ is defined by the fact that its independent sets are the complements of the spanning sets of $M_{A}\left(\mathcal{I}_{P}(C)\right)$. That is, $X$ is an independent set of $M_{A}\left(\mathcal{I}_{P}(C)\right)^{*}$ if and only if the columns of $\mathcal{I}_{P}(C)$ corresponding to elements of $Y=$ $V\left(\mathcal{I}_{P}(C)\right)-X$ span the column space $W$ of $\mathcal{I}_{P}(C)$.

If $\rho\left(\mathcal{I}_{P}(C)-X\right)=\rho\left(\mathcal{I}_{P}(C)\right)$ then $Y$ has $\rho\left(\mathcal{I}_{P}(C)\right)$ independent columns, and these must certainly span $W$.

Conversely, if $W$ is spanned by the columns of $\mathcal{I}_{P}(C)$ corresponding to elements of $Y$ then there must be a subset $B \subseteq Y$ such that the columns of $\mathcal{I}_{P}(C)$ corresponding to elements of $B$ constitute a basis of $W$. As $\mathcal{I}_{P}(C)$ is symmetric, the strong principal minor theorem of Kodiyalam, Lam and Swan [24] (see Theorem 40 below) tells us that the principal submatrix of $\mathcal{I}_{P}(C)$ corresponding to $B$ is nonsingular. This principal submatrix is a submatrix of $\mathcal{I}_{P}(C)-X$, so $\rho\left(\mathcal{I}_{P}(C)-X\right) \geqslant|B|=\rho\left(\mathcal{I}_{P}(C)\right)$. The opposite inequality is obvious, so $\rho\left(\mathcal{I}_{P}(C)-X\right)=\rho\left(\mathcal{I}_{P}(C)\right)$.

As mentioned in the introduction, the duality between touch-graphs and the adjacency matroids of looped circle graphs motivates many properties of general adjacency matroids. For instance, suppose $C$ is an Euler system of a 4-regular graph $F, P$ is a circuit partition compatible with $C$, and $v \in V(F)$. Then Figure 1 makes it clear that if $P^{\prime}$ is the circuit partition obtained from $P$ by changing the transition at $v$ to the other transition that does not appear in $C$, then $v$ is a loop in at least one of the graphs $T \operatorname{ch}(P), T \operatorname{ch}\left(P^{\prime}\right)$. It follows that $v$ is a loop of at least one of the matroids $M_{A}\left(\mathcal{I}_{P}(C)\right)^{*}, M_{A}\left(\mathcal{I}_{P^{\prime}}(C)\right)^{*}$. Thus touch-graphs motivate Proposition 6.

Similarly, touch-graphs motivate Theorem 21 of the introduction. Let $F$ be a 4-regular graph with a vertex $v$ and an Euler system $C$. Kotzig [25] observed that $F$ also has an Euler system $C * v$, which involves the same transition as $C$ at every vertex other than $v$, and at $v$ involves the transition that is orientation-inconsistent with respect to $C$. Let $P$ be a circuit partition of $F$ that is compatible with $C$. Then the following observations explain the duals of the three assertions of Theorem 21. 1. If $v$ is unlooped in $\mathcal{I}_{P}(C)$ then $P$ involves the transition at $v$ that is consistent with the orientation of $C, P$ is compatible with $C * v$ and $\mathcal{I}_{P}(C * v)$ is the local complement $\mathcal{I}_{P}(C)^{v}$. Then $M_{A}\left(\mathcal{I}_{P}(C)\right)=M_{A}\left(\mathcal{I}_{P}(C)^{v}\right)$ because both are dual to the polygon matroid of $T c h(P)$. 2. Suppose $v$ is looped in $\mathcal{I}_{P}(C)$, and let $P^{\prime}$ be the circuit partition obtained from $P$ by changing the transition at $v$ to the one that is orientation-inconsistent with $C * v$. Then $\mathcal{I}_{P}(C)^{v}=\mathcal{I}_{P^{\prime}}(C * v)$. If $v$ is a loop in 
both $T \operatorname{ch}(P)$ and $T \operatorname{ch}\left(P^{\prime}\right)$, then $T \operatorname{ch}(P)=T \operatorname{ch}\left(P^{\prime}\right)$ and hence $M_{A}\left(\mathcal{I}_{P}(C)\right)=M_{A}\left(\mathcal{I}_{P}(C)^{v}\right)$ as both are dual to the polygon matroid of $T \operatorname{ch}(P)=T \operatorname{ch}\left(P^{\prime}\right)$. 3. Suppose $v$ is looped in $\mathcal{I}_{P}(C)$, but not a loop in $T \operatorname{ch}(P)$. Again, let $P^{\prime}$ be the circuit partition obtained from $P$ by changing the transition at $v$ to the one that is orientation-inconsistent with $C * v$. Then $T \operatorname{ch}\left(P^{\prime}\right)$ is the graph obtained from $T \operatorname{ch}(P)$ by contracting $v$ and then attaching a loop at the vertex corresponding to $v$, so the dual of the polygon matroid of $T \operatorname{ch}\left(P^{\prime}\right)$ is isomorphic to the matroid obtained from the dual of the polygon matroid of $T \operatorname{ch}(P)$ by deleting $v$ and replacing it with a coloop.

\section{Theorem 13}

Let $G$ be any graph with no isolated, unlooped vertex. Then a 4-regular graph $F$ with a distinguished circuit partition $P$ may be constructed from $G$ in two steps, as follows.

Step 1. For each vertex $v \in V(G)$, list the incident non-loop edges as $e_{1}^{v}, \ldots, e_{d_{v}}^{v}$ in some order. Then construct a 4-regular graph $H$ with a vertex for each non-loop edge of $G$, in such a way that for each $v \in V(G), H$ has $d_{v}$ edges; the $i^{t h}$ of these edges connects the vertex corresponding to $e_{i}^{v}$ to the vertex corresponding to $e_{i+1}^{v}$ (with subindices considered modulo $\left.d_{v}\right)$. $H$ has a distinguished circuit partition whose elements correspond to the non-isolated vertices of $G$.

Step 2. Suppose $G$ has $\ell$ loops. If $\ell=0$ then let $F=H$, and let $P$ be the distinguished circuit partition. Otherwise, let $H_{0}=H$ and list the loops of $G$ as $e_{1}, \ldots, e_{\ell}$ in some order. Suppose $1 \leqslant i \leqslant \ell$ and $H_{i-1}$ has been constructed with a distinguished circuit partition whose circuits correspond to some of the vertices of $G$. If the vertex of $G$ incident on $e_{i}$ does not correspond to a distinguished circuit of $H_{i-1}$, then $H_{i}$ is obtained from $H_{i-1}$ by adjoining a new vertex with a "figure eight" on it; that is, a single distinguished circuit consisting of two loops. The new distinguished circuit corresponds to the vertex of $G$ incident on $e_{i}$, and the new vertex corresponds to $e_{i}$. If the vertex of $G$ incident on $e_{i}$ does correspond to a distinguished circuit of $H_{i-1}$, then $H_{i}$ is obtained from $H_{i-1}$ by inserting a looped vertex "in the middle" of some edge of this circuit. The original edge disappears, and the corresponding distinguished circuit of $H_{i}$ includes the three new edges and all the other edges of the original distinguished circuit of $H_{i-1}$.

At the end of this process we have obtained $H_{\ell}=F$, with a distinguished circuit partition $P$ such that $T \operatorname{ch}(P) \cong G$.

The construction is illustrated in Figure 2, where distinguished circuit partitions are indicated by the convention that when following a distinguished circuit through a vertex, one does not change the "edge style" (bold, dashed or plain). The "edge style" may change in the middle of an edge, though.

Note that there is considerable freedom in the construction, both in choosing the edgeorders $e_{1}^{v}, \ldots, e_{d_{v}}^{v}$ and in locating the non-isolated, looped vertices introduced in Step 2. Consequently the resulting 4-regular graph is far from unique. In Figure 2, for example, $F$ is a planar graph with two pairs of parallel non-loop edges, and $F^{\prime}$ is a non-planar graph with only one pair of parallel non-loop edges. The fact that $F$ is planar and $F^{\prime}$ is non-planar hints at the fact that when we order the non-loop edges incident at each 

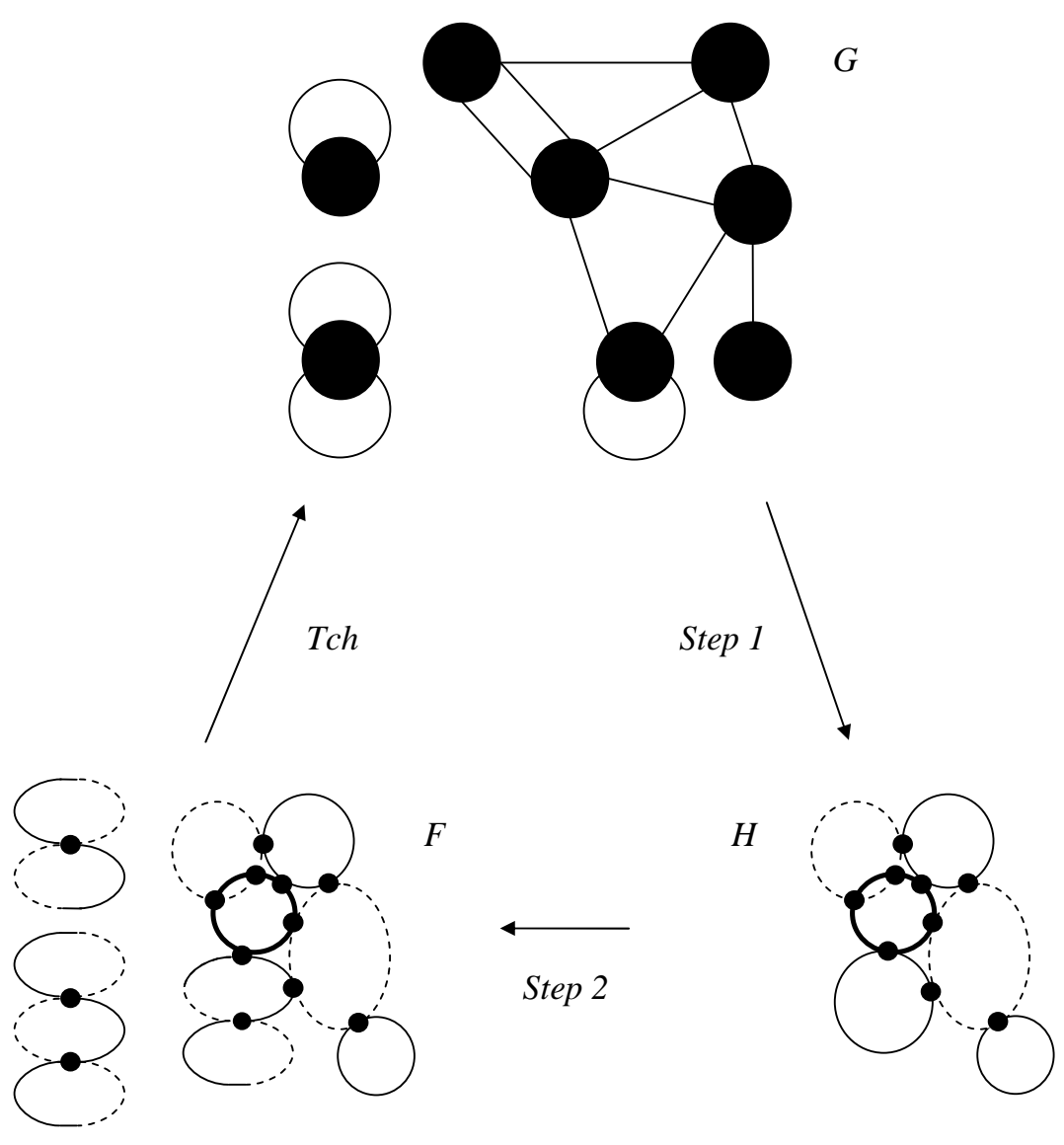

or

or
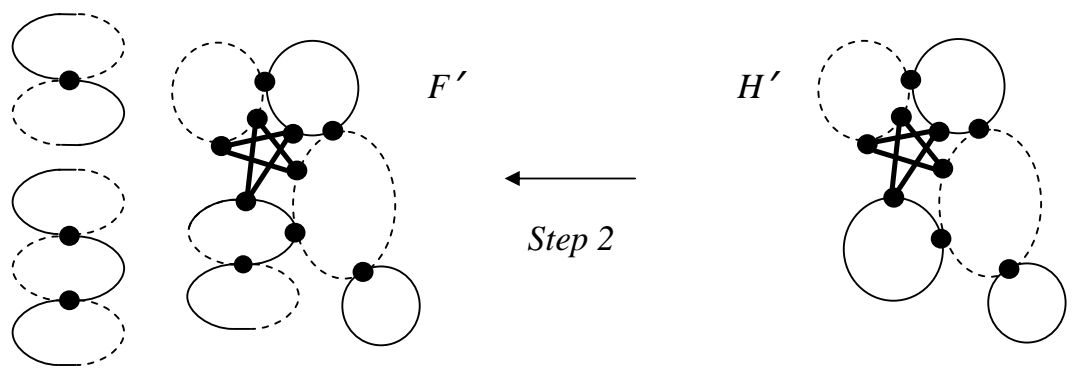

Figure 2: Bold, dashed and plain "edge styles" indicate distinguished circuit partitions $P$ and $P^{\prime}$ with $T \operatorname{ch}(P)$ and $T \operatorname{ch}\left(P^{\prime}\right)$ isomorphic to $G$. 
vertex of $G$, we have chosen a rotation system for the graph obtained from $G$ by removing all loops; equivalently, we have imbedded this graph on some orientable surface. The resulting 4-regular graph $H$ is the medial graph of this imbedded graph.

\section{Local complements and matroid minors}

The reader familiar with the interlace polynomials of Arratia, Bollobás and Sorkin [2, 3, 4] will recognize some of the concepts and notation that appear in our discussion of adjacency matroids, but it is important to keep a significant difference in mind: The interlace polynomials of $G$ are related to principal submatrices of $\mathcal{A}(G)$, i.e., square submatrices obtained from $\mathcal{A}(G)$ by removing some columns and the corresponding rows. The adjacency matroid of $G$, instead, is related to rectangular submatrices obtained by removing only columns from $\mathcal{A}(G)$.

\subsection{Theorems 17 and 20}

Suppose $v \in V(G)$; let

$$
\mathcal{A}(G)=\left(\begin{array}{ccc}
* & \mathbf{1} & \mathbf{0} \\
\mathbf{1} & A & B \\
\mathbf{0} & C & D
\end{array}\right) \text { and } \mathcal{A}\left(G^{v}\right)=\left(\begin{array}{ccc}
* & \mathbf{1} & \mathbf{0} \\
\mathbf{1} & A^{c} & B \\
\mathbf{0} & C & D
\end{array}\right) .
$$

Here bold numerals indicate rows and columns with all entries equal, the first row and column correspond to $v, *$ is 0 or 1 according to the loop status of $v$, and $A^{c}$ is the matrix obtained by toggling all the entries of $A$. To prove Theorem 20, observe that elementary row operations transform

$$
\left(\begin{array}{ll}
\mathbf{1} & \mathbf{0} \\
A & B \\
C & D
\end{array}\right) \text { into }\left(\begin{array}{cc}
\mathbf{1} & \mathbf{0} \\
A^{c} & B \\
C & D
\end{array}\right)
$$

It follows that if $\kappa$ is a column vector then

$$
\left(\begin{array}{cc}
\mathbf{1} & \mathbf{0} \\
A & B \\
C & D
\end{array}\right) \cdot \kappa=\mathbf{0} \text { if and only if }\left(\begin{array}{cc}
\mathbf{1} & \mathbf{0} \\
A^{c} & B \\
C & D
\end{array}\right) \cdot \kappa=\mathbf{0} .
$$

That is, these two matrices have the same right nullspace. It follows that $Z\left(M_{A}(G)-v\right)$ $=Z\left(M_{A}\left(G^{v}\right)-v\right)$, and hence $M_{A}(G)-v=M_{A}\left(G^{v}\right)-v$, as asserted by Theorem 20 .

Theorem 17 follows from another calculation using elementary row operations. Matrices of the forms

$$
\left(\begin{array}{lll}
1 & \mathbf{1} & \mathbf{0} \\
\mathbf{1} & A & B \\
\mathbf{0} & C & D
\end{array}\right),\left(\begin{array}{ccc}
1 & \mathbf{1} & \mathbf{0} \\
\mathbf{0} & A^{c} & B \\
\mathbf{0} & C & D
\end{array}\right) \text { and }\left(\begin{array}{cc}
A^{c} & B \\
C & D
\end{array}\right)
$$

have the same $G F(2)$-nullity, so if $v$ is looped and $v \notin S \subseteq V(G)$ then $S \cup\{v\}$ contains a circuit of $M_{A}(G)$ if and only if $S$ contains a circuit of $M_{A}\left(G^{v}-v\right)$. It follows that if $v$ is looped, then $M_{A}(G) / v=M_{A}\left(G^{v}-v\right)$. 


\subsection{Theorem 19 and the strong principal minor theorem}

We turn now to Theorem 19. Suppose $v$ is not a coloop of $M_{A}(G)$. Then $\mathcal{A}(G)$ is a symmetric matrix of the form

$$
\left(\begin{array}{ccc}
* & \mathbf{1} & \mathbf{0} \\
\mathbf{1} & A & B \\
\mathbf{0} & C & D
\end{array}\right)
$$

with the first column corresponding to $v$ and equal to the sum of certain other columns. It follows that

$$
\left(\begin{array}{cc}
\mathbf{1} & \mathbf{0} \\
A & B \\
C & D
\end{array}\right) \text { and }\left(\begin{array}{cc}
A & B \\
C & D
\end{array}\right)
$$

are related through elementary row operations, so these two matrices have the same right nullspace: $Z\left(M_{A}(G)-v\right)=Z\left(M_{A}(G-v)\right)$. Consequently $M_{A}(G)-v=M_{A}(G-v)$, as asserted by Theorem 19 .

By the way, note that this argument still applies if $v$ is a coloop, provided that $v$ is not a coloop of the adjacency matroid of the graph obtained from $G$ by toggling the loop status of $v$.

Theorem 19 is equivalent to the following special case of the strong principal minor theorem of Kodiyalam, Lam and Swan [24].

Theorem 40. Let $A$ be a symmetric $n \times n$ matrix with entries in $G F(2)$ and let $S$ be a subset of $\{1, \ldots, n\}$, of size $r=\operatorname{rank}(A)$. Then the columns of $A$ corresponding to elements of $S$ are linearly independent if and only if the principal submatrix of $A$ corresponding to $S$ is nonsingular.

Proof. If the principal submatrix of $A$ corresponding to $S$ is nonsingular then its columns must be linearly independent. Obviously then the corresponding columns of $A$, which are obtained from the columns of the principal submatrix by inserting rows corresponding to elements of $\{1, \ldots, n\} \backslash S$, must also be linearly independent.

The interesting part of the theorem is the converse: if the columns of $A$ corresponding to elements of $S$ form an $n \times r$ matrix of rank $r$, then the $r \times r$ submatrix obtained by removing the rows corresponding to elements of $\{1, \ldots, n\} \backslash S$ is also of rank $r$. The proof is simple: Let $A^{\prime}$ be the $n \times r$ submatrix of $A$ that includes only the columns with indices from $S$; by hypothesis, $A$ and $A^{\prime}$ have the same column space. If $i \in\{1, \ldots, n\} \backslash S$ then the $i$ th column of $A$ must be the sum of some columns with indices from $S$, and by symmetry the $i$ th row of $A$ must be the sum of some rows with indices from $S$. Consequently the same is true of the $i$ th row of $A^{\prime}$. It follows that removing the $i$ th row of $A^{\prime}$ for every $i \notin S$ yields an $r \times r$ submatrix with the same row space as $A^{\prime}$, and hence the same rank as $A$. 


\subsection{Theorems 18 and 21}

To prove part 1 of Theorem 21, suppose $v \in V(G)$ is unlooped. Let

$$
\mathcal{A}(G)=\left(\begin{array}{lll}
0 & \mathbf{1} & \mathbf{0} \\
\mathbf{1} & A & B \\
\mathbf{0} & C & D
\end{array}\right) \text { and } \mathcal{A}\left(G^{v}\right)=\left(\begin{array}{ccc}
0 & \mathbf{1} & \mathbf{0} \\
\mathbf{1} & A^{c} & B \\
\mathbf{0} & C & D
\end{array}\right)
$$

Elementary row operations transform $\mathcal{A}(G)$ into $\mathcal{A}\left(G^{c}\right)$, so these matrices have the same right nullspace. It follows that $Z\left(M_{A}(G)\right)=Z\left(M_{A}\left(G^{v}\right)\right)$.

We are now ready to prove all three parts of Theorem 18. If $v$ is unlooped and isolated then $v$ is a loop in $M_{A}(G)$, so $M_{A}(G) / v=M_{A}(G)-v$; Theorem 19 tells us that $M_{A}(G)-v$ $=M_{A}(G-v)$. If $v$ and $w$ are unlooped neighbors then part 1 of Theorem 21 tells us that $M_{A}(G)=M_{A}\left(G^{w}\right) ; v$ is looped in $G^{w}$, so Theorem 17 tells us that $M_{A}\left(G^{w}\right) / v=$ $M_{A}\left(\left(G^{w}\right)^{v}-v\right)$. Finally, if $v$ is unlooped and $w$ is a looped neighbor of $v$ in $G$ then part 1 of Theorem 21 tells us that $M_{A}(G)=M_{A}\left(G^{v}\right) ; w$ is an unlooped neighbor of $v$ in $G^{v}$, so the preceding sentence tells us that $M_{A}(G) / v=M_{A}\left(G^{v}\right) / v=M_{A}\left(\left(\left(G^{v}\right)^{w}\right)^{v}-v\right)$.

Turning to part 2 of Theorem 21, suppose a looped vertex $v$ is a coloop of both $M_{A}(G)$ and $M_{A}\left(G^{v}\right)$. Then $\mathcal{C}\left(M_{A}(G)\right)=\mathcal{C}\left(M_{A}(G)-v\right)$ and $\mathcal{C}\left(M_{A}\left(G^{v}\right)\right)=\mathcal{C}\left(M_{A}\left(G^{v}\right)-v\right)$. Theorem 20 tells us that $M_{A}(G)-v=M_{A}\left(G^{v}\right)-v$, so $M_{A}(G)=M_{A}\left(G^{v}\right)$.

Part 3 of Theorem 21 involves the following.

Lemma 41. Suppose $v \in V(G)$. Then $v$ is not a coloop of $M_{A}(G)$ if and only if the three matrices

$$
\mathcal{A}(G)=\left(\begin{array}{ccc}
* & \mathbf{1} & \mathbf{0} \\
\mathbf{1} & A & B \\
\mathbf{0} & C & D
\end{array}\right),\left(\begin{array}{ll}
\mathbf{1} & \mathbf{0} \\
A & B \\
C & D
\end{array}\right) \text { and }\left(\begin{array}{cc}
\mathbf{0} & \mathbf{0} \\
A & B \\
C & D
\end{array}\right)
$$

have the same rank over $G F(2)$. (Here the first row and column of $\mathcal{A}(G)$ correspond to v.)

Proof. If the three matrices have the same rank then in particular, the first two have the same rank. Consequently the first column of $\mathcal{A}(G)$ must equal the sum of certain other columns; hence there is a circuit of $M_{A}(G)$ that contains $v$. Conversely, if $v$ is not a coloop of $M_{A}(G)$ then the column of $\mathcal{A}(G)$ corresponding to $v$ must be the sum of the columns corresponding to some subset $S_{v} \subseteq V(G) \backslash\{v\}$. By symmetry, the sum of the rows corresponding to $S_{v}$ must equal the first row of $\mathcal{A}(G)$.

Suppose now that $v$ is a looped non-coloop of $M_{A}(G)$. The set $S_{v}$ must include an odd number of columns of $A$, to yield the diagonal entry $*=1$ in the column of $\mathcal{A}(G)$ corresponding to $v$. Replacing $A$ with $A^{c}$ toggles an odd number of summands in each row of $A$, so the sum of the columns of

$$
\mathcal{A}\left(G^{v}\right)=\left(\begin{array}{ccc}
1 & \mathbf{1} & \mathbf{0} \\
\mathbf{1} & A^{c} & B \\
\mathbf{0} & C & D
\end{array}\right)
$$


corresponding to $S_{v}$ must be the column vector

$$
\left(\begin{array}{l}
1 \\
0 \\
0
\end{array}\right)
$$

It follows that the $G F(2)$-ranks of

$$
\left(\begin{array}{cc}
\mathbf{1} & \mathbf{0} \\
A^{c} & B \\
C & D
\end{array}\right) \text { and }\left(\begin{array}{ccc}
1 & \mathbf{0} & \mathbf{0} \\
\mathbf{0} & A^{c} & B \\
\mathbf{0} & C & D
\end{array}\right)
$$

are the same. According to Lemma 41, $v$ cannot be a non-coloop of $M_{A}\left(G^{v}\right)$.

By the way, the same argument shows that removing the loop from $v$ cannot produce a non-coloop in the adjacency matroid of the resulting graph. That is, in the terminology of Section $4 v$ is a triple coloop of $M_{A}\left(G^{v}\right)$.

To complete the proof of part 3 of Theorem 21, note that the fact that $v$ is a coloop of $M_{A}\left(G^{v}\right)$ implies that $\mathcal{C}\left(M_{A}\left(G^{v}\right)\right)=\mathcal{C}\left(M_{A}\left(G^{v}\right)-v\right)$. Theorem 20 tells us that $\mathcal{C}\left(M_{A}\left(G^{v}\right)-\right.$ $v)=\mathcal{C}\left(M_{A}(G)-v\right)$, and the fact that $v$ is not a coloop of $M_{A}(G)$ implies that $\mathcal{C}\left(M_{A}(G)-\right.$ $v)$ is a proper subset of $\mathcal{C}\left(M_{A}(G)\right)$. It follows that $\left|\mathcal{C}\left(M_{A}\left(G^{v}\right)\right)\right|<\left|\mathcal{C}\left(M_{A}(G)\right)\right|$, and consequently $M_{A}\left(G^{v}\right) \not M_{A}(G)$. The equality $M_{A}\left(G^{v}\right)=\left(M_{A}\left(G^{v}\right)-v\right) \oplus U_{1,1}(\{v\})$ follows immediately from the fact that $v$ is a coloop of $M_{A}\left(G^{v}\right)$, and this equality implies $M_{A}\left(G^{v}\right)=\left(M_{A}(G)-v\right) \oplus U_{1,1}(\{v\})$ by Theorem 20 .

\section{Theorem 23 and triple coloops}

Theorem 23 is essentially a result about the nullspaces of $\mathcal{A}(G(v)), \mathcal{A}(G(v, \ell))$ and $\mathcal{A}(G(v, \ell i))$. With a convenient order on the vertices of $G$, these three matrices are

$$
\left(\begin{array}{lll}
0 & \mathbf{1} & \mathbf{0} \\
\mathbf{1} & A & B \\
\mathbf{0} & C & D
\end{array}\right),\left(\begin{array}{ccc}
1 & \mathbf{1} & \mathbf{0} \\
\mathbf{1} & A & B \\
\mathbf{0} & C & D
\end{array}\right) \text { and }\left(\begin{array}{ccc}
1 & \mathbf{0} & \mathbf{0} \\
\mathbf{0} & A & B \\
\mathbf{0} & C & D
\end{array}\right)
$$

respectively. Theorem 23 asserts that two of the nullspaces are the same, say of dimension $\nu$; the different nullspace contains them, and its dimension is $\nu+1$. A proof of this statement is given in [31].

Observe that no element of the nullspace of the right-hand matrix could possibly have a nonzero first coordinate. Consequently $v$ does not appear in any circuit of $M_{A}(G(v, \ell i))$; that is, $v$ is a coloop of $M_{A}(G(v, \ell i))$, as noted in Proposition 24 of the introduction. In the special case that $M_{A}(G(v, \ell i))$ has a larger cycle space than $M_{A}(G(v))=M_{A}(G(v, \ell))$, it follows that $v$ must also be a coloop of $M_{A}(G(v))$ and $M_{A}(G(v, \ell))$. On the other hand, if $M_{A}(G(v))$ or $M_{A}(G(v, \ell))$ has a larger cycle space than $M_{A}(G(v, \ell i))$ then either the matrix displayed on the left or the matrix displayed in the center has a larger nullspace than the one on the right. Clearly any vector in either of these two nullspaces that is not in 
the nullspace of the right-hand matrix must have a nonzero first coordinate; consequently $v$ is not a coloop of the corresponding matroid. We deduce the following sharpened form of Proposition 6.

Corollary 42. If $v \in V(G)$ then $v$ is a coloop of $M_{A}(G(v, \ell i))$ and at least one of the adjacency matroids $M_{A}(G(v)), M_{A}(G(v, \ell))$. It is a coloop of all three if and only if

$$
Z\left(M_{A}(G(v))\right)=Z\left(M_{A}(G(v, \ell)) \subset Z\left(M_{A}(G(v, \ell i))\right) .\right.
$$

The special case in which $v$ is a coloop of all three matroids is important enough to merit a special name.

Definition 43. A vertex $v \in V(G)$ is a triple coloop of $M_{A}(G)$ if it is a coloop of $M_{A}(G(v)), M_{A}(G(v, \ell))$, and $M_{A}(G(v, \ell i))$.

Note that the nomenclature is imprecise; although a triple coloop of $M_{A}(G)$ is certainly a coloop of $M_{A}(G)$, it is not the matroid structure of $M_{A}(G)$ that determines whether or not a vertex is a triple coloop. We prefer this imprecise nomenclature over the alternative " $v$ is a triple coloop of $G$ " because that would also be confusing; a coloop (isthmus) of $G$ is an edge, not a vertex.

Using this notion, Theorems 19 and 21 may be sharpened as follows.

Theorem 44. 1. If $v \in V(G)$ is unlooped then $M_{A}\left(G^{v}\right)=M_{A}(G)$.

2. If a looped vertex $v \in V(G)$ is a coloop of both $M_{A}(G)$ and $M_{A}\left(G^{v}\right)$, then $M_{A}\left(G^{v}\right)$ $=M_{A}(G)$ and $v$ is not a triple coloop of $M_{A}\left(G^{v}\right)$ or $M_{A}(G)$.

3. If $v \in V(G)$ is looped and not a coloop of one of $M_{A}(G), M_{A}\left(G^{v}\right)$, then $v$ is a triple coloop of the other and $M_{A}\left(G^{v}\right) \not M_{A}(G)$. More specifically, if $\left\{M_{A}(G), M_{A}\left(G^{v}\right)\right\}=$ $\left\{M_{1}, M_{2}\right\}$ with $v$ not a coloop of $M_{1}$, then $v$ is a triple coloop of $M_{2}$ and $M_{2}=\left(M_{1}-\right.$ $v) \oplus U_{1,1}(\{v\})$.

Theorem 45. If $v \in V(G)$ is not a triple coloop of $M_{A}(G)$, then $M_{A}(G)-v=M_{A}(G-v)$.

Proof. Part 1 of Theorem 44 is the same as part 1 of Theorem 21. The proofs of Theorem 45 and part 3 of Theorem 44 are indicated in the preceding section; both are introduced with the phrase "by the way."

It remains to consider part 2 of Theorem 44. Suppose a looped vertex $v \in V(G)$ is a triple coloop of $G$, and let

$$
\mathcal{A}(G)=\mathcal{A}(G(v, \ell))=\left(\begin{array}{lll}
1 & \mathbf{1} & \mathbf{0} \\
\mathbf{1} & A & B \\
\mathbf{0} & C & D
\end{array}\right) .
$$

According to Theorem 23 and Corollary 42,

$$
\nu(\mathcal{A}(G(v)))=\nu(\mathcal{A}(G(v, \ell)))=\nu(\mathcal{A}(G(v, \ell i)))-1,
$$


where $\nu$ denotes the $G F(2)$-nullity. Observe that elementary row and column operations transform $\mathcal{A}(G(v, \ell))$ into

$$
\left(\begin{array}{ccc}
1 & \mathbf{0} & \mathbf{0} \\
\mathbf{0} & A^{c} & B \\
\mathbf{0} & C & D
\end{array}\right)
$$

which is $\mathcal{A}\left(\left(G^{v}(v, \ell i)\right)\right.$. It follows that $\nu(\mathcal{A}(G(v, \ell)))=\nu\left(\mathcal{A}\left(G^{v}(v, \ell i)\right)\right)$. Also, elementary row operations transform

$$
\mathcal{A}(G(v))=\left(\begin{array}{lll}
0 & \mathbf{1} & \mathbf{0} \\
\mathbf{1} & A & B \\
\mathbf{0} & C & D
\end{array}\right) \text { into }\left(\begin{array}{ccc}
0 & \mathbf{1} & \mathbf{0} \\
\mathbf{1} & A^{c} & B \\
\mathbf{0} & C & D
\end{array}\right)=\mathcal{A}\left(G^{v}(v)\right)
$$

so $\nu(\mathcal{A}(G(v)))=\nu\left(\mathcal{A}\left(G^{v}(v)\right)\right)$; hence $\nu\left(\mathcal{A}\left(G^{v}(v, \ell i)\right)\right)=\nu\left(\mathcal{A}\left(G^{v}(v)\right)\right)$. According to Theorem 23 and Corollary 42, this equality implies that $v$ is not a coloop of $M_{A}\left(G^{v}(v, \ell)\right)=$ $M_{A}\left(G^{v}\right)$.

\section{The principal vertex tripartition}

Combining various results above, we see that if $v \in V(G)$ then the relationships among the six adjacency matroids $M_{A}(G(v)), M_{A}(G(v, \ell)), M_{A}\left(G^{v}(v)\right), M_{A}(G(v, \ell i)), M_{A}\left(G^{v}(v, \ell)\right)$, $M_{A}\left(G^{v}(v, \ell i)\right)$ must fall into one of three cases.

Case 1. Suppose $v$ is not a coloop of $M_{A}\left(G^{v}(v, \ell)\right)$. According to part 3 of Theorem $44, v$ is a triple coloop of $M_{A}(G(v, \ell))$. Theorem 23 tells us that

$$
Z\left(M_{A}\left(G^{v}(v)\right)\right)=Z\left(M_{A}\left(G^{v}(v, \ell i)\right)\right) \subset Z\left(M_{A}\left(G^{v}(v, \ell)\right)\right)
$$

and

$$
Z\left(M_{A}(G(v))\right)=Z\left(M_{A}(G(v, \ell))\right) \subset Z\left(M_{A}(G(v, \ell i))\right) .
$$

Part 1 of Theorem 21 tells us that $M_{A}\left(G^{v}(v)\right)=M_{A}(G(v)) . \quad M_{A}(G(v, \ell i))$ has $v$ as a coloop, so the fact that $v$ is not a coloop of $M_{A}\left(G^{v}(v, \ell)\right)$ implies that $M_{A}(G(v, \ell i)) \neq$ $M_{A}\left(G^{v}(v, \ell)\right)$. All in all, we have the following: $Z\left(M_{A}(G(v, \ell i))\right)$ and $Z\left(M_{A}\left(G^{v}(v, \ell)\right)\right)$ are distinct nontrivial subspaces of $2^{V(G)}$ with the same dimension, say $\nu+1$; their intersection is of dimension $\nu$, and

$$
\begin{aligned}
Z\left(M_{A}\left(G^{v}(v, \ell i)\right)\right) & =Z\left(M_{A}\left(G^{v}(v)\right)\right)=Z\left(M_{A}(G(v))\right)=Z\left(M_{A}(G(v, \ell))\right. \\
& =Z\left(M_{A}(G(v, \ell i))\right) \cap Z\left(M_{A}\left(G^{v}(v, \ell)\right)\right) .
\end{aligned}
$$

The equality $M_{A}\left(G^{v}(v, \ell i)\right)=\left(M_{A}\left(G^{v}(v, \ell)\right)-v\right) \oplus U_{1,1}(\{v\})$ follows from Theorem 19 and Proposition 24, and $M_{A}(G(v, \ell i))=\left(M_{A}\left(G^{v}(v, \ell)\right) / v\right) \oplus U_{1,1}(\{v\})$ follows from Proposition 24.

Case 2. Suppose $v$ is not a coloop of $M_{A}(G(v, \ell))$. The discussion proceeds as in case 1 , with $G$ and $G^{v}$ interchanged.

Case 3. Suppose $v$ is a coloop of both $M_{A}(G(v, \ell))$ and $M_{A}\left(G^{v}(v, \ell)\right)$; then parts 1 and 2 of Theorem 44 tell us that $M_{A}(G(v))=M_{A}\left(G^{v}(v)\right), M_{A}(G(v, \ell))=M_{A}\left(G^{v}(v, \ell)\right)$, and 
$v$ is not a triple coloop of $M_{A}(G(v, \ell))$ or $M_{A}\left(G^{v}(v, \ell)\right)$. Then Theorem 23 and Corollary 42 tell us that

$$
\begin{aligned}
Z\left(M_{A}(G(v, \ell))\right) & =Z\left(M_{A}\left(G^{v}(v, \ell)\right)\right)=Z\left(M_{A}(G(v, \ell i))\right)=Z\left(M_{A}\left(G^{v}(v, \ell i)\right)\right) \\
& \subset Z\left(M_{A}(G(v))\right)=Z\left(M_{A}\left(G^{v}(v)\right)\right)
\end{aligned}
$$

with the dimension of the larger subspace 1 more than the dimension of the smaller. The equality $M_{A}(G(v, \ell i))=\left(M_{A}(G(v))-v\right) \oplus U_{1,1}(\{v\})$ follows from Theorem 19 and Proposition 24.

To complete the proof of Theorem 25, we must verify the assertion that if $G^{v}-v$ is simple, then $v$ cannot fall under case 1 of the tripartition. Suppose $v \in V(G)$ falls under case 1 , and let

$$
\mathcal{A}\left(G^{v}(v, \ell)\right)=\left(\begin{array}{ccc}
1 & \mathbf{1} & \mathbf{0} \\
\mathbf{1} & A & B \\
\mathbf{0} & C & D
\end{array}\right)
$$

with the first row and column corresponding to $v$. As $v$ is not a coloop of $M_{A}\left(G^{v}(v, \ell)\right)$, the first column of $\mathcal{A}\left(G^{v}(v, \ell)\right)$ must equal the sum of the columns corresponding to elements of some subset $T \subseteq V(G) \backslash\{v\}$. Consider the submatrix of $\mathcal{A}\left(G^{v}(v, \ell)\right)$ obtained by removing the rows and columns corresponding to vertices not in $T \cup\{v\}$,

$$
\mathcal{A}\left(G^{v}(v, \ell)[T \cup\{v\}]\right)=\left(\begin{array}{ccc}
1 & \mathbf{1} & \mathbf{0} \\
\mathbf{1} & A^{\prime} & B^{\prime} \\
\mathbf{0} & C^{\prime} & D^{\prime}
\end{array}\right) .
$$

The sum of the columns of this matrix is $\mathbf{0}$, so the sum of the entries of the matrix is 0 ; that is, the matrix has an even number of nonzero entries. As the matrix is symmetric, an even number of these nonzero entries occur off the diagonal; consequently an even number occur on the diagonal, so at least one element of $T$ is looped in $G^{v}$.

Essentially the same argument proves that in case 2, at least one element of $T$ is looped in $G$. We should point out that a garbled version of this simple argument appeared in [29], where it was mistakenly understood to imply that there must be at least one looped vertex in $T \cap N(v)$. This need not be the case, as indicated by the third example in the next section. The statements of Lemma 4.4 and Corollary 4.6 of [29] should be corrected by replacing the hypothesis "if $a$ has no looped neighbor" with "if $H-a$ has no looped vertex."

\section{Three examples}

Recall that if $k<n$ then $U_{n, k}$ denotes the $n$-element matroid whose circuits include all the $(k+1)$-element subsets of the ground set. Also, $U_{n, n}$ denotes the free matroid on $n$ elements, i.e., $\mathcal{C}\left(U_{n, n}\right)=\varnothing$.

Let $K_{3}$ be the complete graph with three vertices. Then $M_{A}\left(K_{3}\right) \cong U_{3,2}$. If $v \in$ $V\left(K_{3}\right)$ then $v$ is not a coloop of either $M_{A}\left(K_{3}\right)$ or $M_{A}\left(K_{3}^{v}\right)$; $v$ falls under case 3 of the 

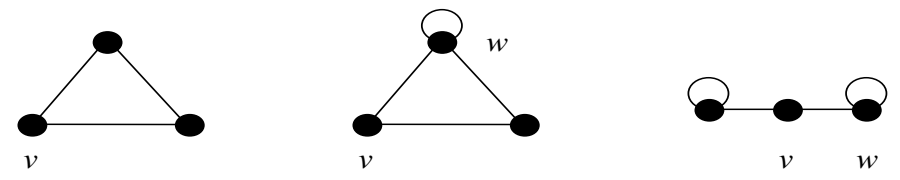

Figure 3: $K_{3}, K_{3 \ell}$ and $P_{3 \ell \ell}$.

principal vertex tripartition. $M_{A}\left(K_{3}(v, \ell)\right)=M_{A}\left(K_{3}(v, \ell i)\right) \cong U_{3,3}, M_{A}\left(K_{3}^{v}\right)=M_{A}\left(K_{3}\right)$, $M_{A}\left(K_{3}-v\right)=M_{A}\left(K_{3}\right)-v \cong U_{2,2}$, and $M_{A}\left(K_{3}\right) / v \cong U_{2,1}$.

Let $K_{3 \ell}$ be the graph obtained from $K_{3}$ by attaching a loop to one vertex. Then $M_{A}\left(K_{3 \ell}\right) \cong U_{3,3}$. If $v$ is one of the unlooped vertices then $v$ is a coloop of $M_{A}\left(K_{3 \ell}\right)$, and $v$ is a triple coloop of $M_{A}\left(K_{3 \ell}^{v}\right) ; v$ falls under case 2 of the principal vertex tripartition. $M_{A}\left(K_{3 \ell}(v, \ell i)\right)=M_{A}\left(K_{3 \ell}\right), M_{A}\left(K_{3 \ell}(v, \ell)\right) \cong U_{1,1} \oplus U_{2,1}$, and $M_{A}\left(K_{3 \ell}-v\right)=M_{A}\left(K_{3 \ell}\right)-v=$ $M_{A}\left(K_{3 \ell}\right) / v \cong U_{2,2}$. If $w$ is the looped vertex then $w$ is a coloop of $M_{A}\left(K_{3 \ell}\right)$ and a coloop of $M_{A}\left(K_{3 \ell}^{w}\right)$, but not a triple coloop of either; $w$ falls under case 3 of the principal vertex tripartition. $M_{A}\left(K_{3 \ell}^{w}\right)=M_{A}\left(K_{3 \ell}(w, \ell i)\right)=M_{A}\left(K_{3 \ell}\right), M_{A}\left(K_{3 \ell}(w)\right) \cong U_{3,2}$, and $M_{A}\left(K_{3 \ell}\right)-w=M_{A}\left(K_{3 \ell}\right) / w=M_{A}\left(K_{3 \ell}-w\right) \cong U_{2,2}$.

Let $P_{3}$ be the path of length three, and $P_{3 \ell \ell}$ the graph obtained from $P_{3}$ by attaching loops at the vertices of degree 1. (Equivalently, $P_{3 \ell \ell}=K_{3}^{v}$.) Then $M_{A}\left(P_{3 \ell \ell}\right) \cong U_{3,2}$. If $v$ is the unlooped vertex then $v$ is not a coloop of $M_{A}\left(P_{3 \ell \ell}\right)$ or $M_{A}\left(P_{3 \ell \ell}^{v}\right) ; v$ falls under case 3 of the principal vertex tripartition. $M_{A}\left(P_{3 \ell \ell}(v, \ell)\right)=M_{A}\left(P_{3 \ell \ell}(v, \ell i)\right) \cong U_{3,3}, M_{A}\left(P_{3 \ell \ell}^{v}\right)=$ $M_{A}\left(P_{3 \ell \ell}\right), M_{A}\left(P_{3 \ell \ell}-v\right)=M_{A}\left(P_{3 \ell \ell}\right)-v \cong U_{2,2}$ and $M_{A}\left(P_{3 \ell \ell}\right) / v \cong U_{2,1}$. If $w$ is one of the looped vertices then $w$ is not a coloop of $M_{A}\left(P_{3 \ell \ell}\right)$, and $w$ is a triple coloop of $M_{A}\left(P_{3 \ell \ell}^{w}\right)$; $w$ falls under case 2 of the principal vertex tripartition. $M_{A}\left(P_{3 \ell \ell}(w)\right)=M_{A}\left(P_{3 \ell \ell}(w, \ell i)\right)$ $=M_{A}\left(P_{3 \ell \ell}^{w}\right) \cong U_{3,3}, M_{A}\left(P_{3 \ell \ell}-w\right)=M_{A}\left(P_{3 \ell \ell}\right)-w \cong U_{2,2}$, and $M_{A}\left(P_{3 \ell \ell}\right) / w \cong U_{2,1}$.

Observe that $K_{3}$ and $P_{3 \ell \ell}$ have isomorphic adjacency matroids, and their principal vertex tripartitions are distinct. On the other hand, $K_{3 \ell}$ and $P_{3 \ell \ell}$ have nonisomorphic adjacency matroids and equivalent principal vertex tripartitions. We deduce Theorem 26 of the introduction.

Note also that both $U_{3,2}$ and $U_{3,3}$ have the property that every permutation of the ground set is a matroid automorphism. Consequently for each of these matroids, all elements of the ground set are equivalent under the principal edge tripartition of Rosenstiehl and Read [28]. In contrast, the elements of $M_{A}\left(K_{3 \ell}\right)$ and $M_{A}\left(P_{3 \ell \ell}\right)$ are not equivalent under the principal vertex tripartition.

\section{Set systems and $\Delta$-matroids}

In this section we briefly summarize a number of definitions and results related to set systems and $\Delta$-matroids. We refer to [9] - [11] and [13] - [15] for detailed discussions. 


\subsection{Set systems}

A set system (over $V$ ) is a tuple $D=(V, \sigma)$ with $V$ a finite set, called the ground set, and $\sigma$ a family of subsets of $V$. We often write $Y \in D$ to mean $Y \in \sigma$. A set system $D$ is called proper if $\sigma \neq \varnothing$, and normal if $\varnothing \in D$. Let $X \subseteq V$. If $D$ is proper, then we define the distance between $X \subseteq V$ and $D$ by $d_{D}(X)=\min (\{|X \Delta Y| \mid Y \in D\})$. Moreover, we let $d_{D}=d_{D}(\varnothing)$, so that $D$ is normal if and only if $d_{D}=0$. We define the restriction of $D$ to $X$ by $D[X]=\left(X, \sigma^{\prime}\right)$ where $\sigma^{\prime}=\{Y \in \sigma \mid Y \subseteq X\}$, and the deletion of $X$ from $D$ by $D-X=D[V-X]$. Let $\min (\sigma)(\max (\sigma)$, resp. $)$ denote the family of minimal (maximal, resp.) sets in $\sigma$ with respect to set inclusion, and let $\min (D)=(V, \min (\sigma))$ $(\max (D)=(V, \max (\sigma))$, resp. $)$ be the corresponding set systems. A set system $D$ is equicardinal if for all $X_{1}, X_{2} \in D,\left|X_{1}\right|=\left|X_{2}\right|$.

Let $D$ again be a set system. For $X \subseteq V$ we define the pivot (also called twist in the literature [9]) by $D * X=(V, \sigma * X)$, where $\sigma * X=\{Y \Delta X \mid Y \in \sigma\}$. Associativity of the symmetric difference implies that $(D * X) * Y=D *(X \Delta Y)$. Also, if $v \in V$ we define the contraction $D / v$ by $D / v=(D *\{v\})-\{v\}$ [11, Property 2.1]. Note that $d_{D}(X)=d_{D * X}$; in particular, $D * X$ is normal if and only if $X \in D$. Also, note that $D * V$ is obtained from $D$ by complementing every set of $D$ with respect to the ground set. Thus, it is easy to see that $\min (D)=\max (D * V) * V$ and $\max (D)=\min (D * V) * V$. For $X \subseteq V$ we define loop complementation by $D+X=\left(V, \sigma^{\prime}\right)$, where $Y \in \sigma^{\prime}$ iff $|\{Z \in D \mid(Y \backslash X) \subseteq Z \subseteq Y\}|$ is odd [13]. In particular, if $v \in V$ then $D+\{v\}=\left(V, \sigma^{\prime}\right)$ with $\sigma^{\prime}=\{Y \mid v \notin Y \in D\} \cup\{Y \cup\{v\} \mid Y \in D$ and $Y \cup\{v\} \notin D\}$. For $X \subseteq V$ we define the dual pivot by $D \bar{*} X=((D+X) * X)+X$. It turns out that $D \bar{*} X=\left(V, \sigma^{\prime}\right)$, where $Y \in \sigma^{\prime}$ iff $|\{Z \in D \mid Y \subseteq Z \subseteq Y \cup X\}|$ is odd. It is easy to verify that $\max (D)=\max (D \bar{*} X)$ for all $X \subseteq V$.

For convenience, we often write $D-\{v\}, D *\{v\}, D \bar{*}\{v\}$ etc. simply as $D-v, D * v$, $D \bar{*} v$ etc. Also, we assume left-associativity of the operations. E.g., $D \bar{*} v * w-v$ denotes $((D \bar{*} v) * w)-v$. Deletion, contraction, pivot, loop complementation, and dual pivot commute with each other when applied to different elements (see [13]). For example, for $v, w \in V$ and $v \neq w, D-v * w=D * w-v, D / v-w=D-w / v, D * v * w=D * w \bar{*} v$ and $D \bar{*} v \bar{*} w=D \bar{*} w \bar{*} v$. Moreover, pivot, loop complementation, and dual pivot are involutions.

Suppose $D=(V, \sigma)$ is a set system, and $v \in V$. Then $\sigma=\sigma^{\prime} \cup \sigma^{\prime \prime}$, where $Y \in \sigma^{\prime}$ (resp. $\left.Y \in \sigma^{\prime \prime}\right)$ iff $v \notin Y \in \sigma$ (resp. $\left.v \in Y \in \sigma\right)$. We define $\widetilde{D^{\sim}} v=\left(V, \sigma^{\prime}\right)$ and $\widetilde{D / v}=\left(V, \sigma^{\prime \prime}\right)$. That is, $D \widetilde{-} v$ is the set system on $V$ that includes the same sets as $D-v$, and $\widetilde{D / v}$ is the set system on $V$ that includes the sets $Y \cup\{v\}$ with $Y \in D / v$.

Theorem 46. Let $D$ be a set system on $V$, and suppose $v \in V$ has the property that $D_{-v}^{\sim}$ is a proper set system. We have

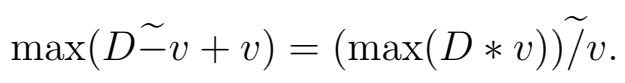

That is, $\max \left(D^{\sim} v+v\right)$ is obtained from $\max (D * v)$ by removing the sets that do not contain $v$. 
Proof. By definition, $D^{\sim} v+v=(V, \sigma)$ where $\sigma=\{Y, Y \cup\{v\} \mid v \notin Y \in D\}$. Consequently all sets in $\max (D \stackrel{\sim}{-v}+v)$ contain $v$; that is, $\max (D \stackrel{\sim}{-v}+v)=\max ((D \stackrel{\sim}{-} v+v) \widetilde{/ v})$. Moreover, the family of sets of $D \stackrel{\sim}{-v}+v$ that include $v$ is $\{Y \cup\{v\} \mid v \notin Y \in D\}$, which is equal to the family of sets of $D * v$ that include $v$. That is, $(D \stackrel{\sim}{-v}+v) \widetilde{/ v}=(D * v) \widetilde{/ v}$; it follows immediately that $\max \left(\left(D^{\sim} \tilde{u} v+v\right) \widetilde{/ v}\right)=\max ((D * v) \widetilde{/ v})$. The equality $(\max ((D * v)) \widetilde{/ v}=$ $\max ((D * v) \widetilde{/} v)$ is obvious; the maximal elements of $D * v$ that contain $v$ are the elements of $D * v$ that are maximal among those that contain $v$.

Theorem 46 is the first of several results that extend properties of adjacency matroids to general set systems or $\Delta$-matroids. As we discuss in Theorem 59 below, this theorem extends part of Proposition 24 of the introduction.

\section{$9.2 \Delta$-matroids}

As mentioned in the introduction, a delta-matroid ( $\Delta$-matroid for short) is a proper set system $D$ that satisfies the symmetric exchange axiom: For all $X, Y \in D$ and all $u \in X \Delta Y, X \Delta\{u\} \in D$ or there is a $v \in X \Delta Y$ with $v \neq u$ such that $X \Delta\{u, v\} \in D$ (or both) [9]. Clearly if $D$ is a $\Delta$-matroid then $D * X$ is a $\Delta$-matroid for every $X \subseteq V$. Also, a proper set system $D$ is a $\Delta$-matroid if and only if for each $X \subseteq V, \min (D * X)$ is equicardinal (see [14]). Equivalently, $D$ is a $\Delta$-matroid if and only if for each $X \subseteq V$, $\max (D * X)$ is equicardinal. Moreover, if $D$ is a $\Delta$-matroid and $v \in V$ then $D-v$ is a $\Delta$-matroid if and only if $D-v$ is proper.

However $D \bar{*} v$ may be a proper set system without being a $\Delta$-matroid. As in $[13$, Example 10], let $V$ be a finite set with $|V| \geqslant 3$, and consider the $\Delta$-matroid $D=(V, \sigma)$ with $\sigma=2^{V} \backslash\{\varnothing\}$. Then it is easy to see that the symmetric exchange axiom does not hold for $D \bar{*} V=(V,\{\varnothing, V\})$.

If we assume a matroid $M$ is described by its family of bases, i.e., $M$ is the set system $(V, B)$ where $B$ is the set of bases of $M$, then it is shown in [10, Proposition 3] that a matroid $M$ is precisely an equicardinal $\Delta$-matroid. Moreover, a proper set system $D$ is a $\Delta$-matroid if and only if for each $X \subseteq V, \max (D * X)$ is a matroid [11, Property 4.1]. Note that for a matroid $M$ (described by its family of bases), $M * V$ is the dual matroid of $M$. Hence, $D$ is a $\Delta$-matroid if and only if for each $X \subseteq V, \min (D * X)$ is a matroid. Clearly for any $\Delta$-matroid $D, r(\min (D))=d_{D}$ and $\nu(\max (D))=d_{D}(V)$, where $r$ and $\nu$ denote the rank and nullity of a matroid respectively. The deletion operation of $\Delta$ matroids coincides with the deletion operation of matroids only for non-coloops. Also, the contraction operation of $\Delta$-matroids coincides with the contraction operation of matroids only for non-loops. Fortunately, as deletion and contraction for matroids coincide for both loops and coloops, matroid-deletion of a coloop is equal to $\Delta$-matroid-contraction of that element, and matroid-contraction of a loop is equal to $\Delta$-matroid-deletion of that element.

We will need Theorem 5.5 from [14] (the original formulation is in terms of rank rather than nullity). 
Proposition 47. Let $D$ be a $\Delta$-matroid, and suppose $v \in V$ has the property that $D+v$ is also a $\Delta$-matroid. Then $\max (D), \max (D * v)$, and $\max (D+v)$ are matroids such that precisely two of the three are equal, to say $D_{1}$. Moreover the third, $D_{2}$, has $\left(D_{2}-v\right) \oplus$ $U_{1,1}(\{v\})=D_{1}$ and $\nu\left(D_{2}\right)=\nu\left(D_{1}\right)+1$.

Note that consequently, $\nu(\max (D))=\nu(\max (D * v))$ if and only if $\max (D)=\max (D *$

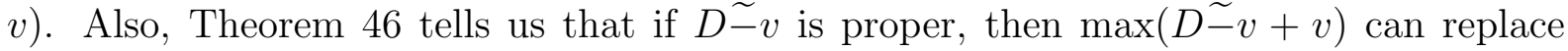
$\max (D * v)$ in Proposition 47: if $\max (D * v)=D_{1}$ then $v$ is a coloop of $\max (D *$ $v)=(\max (D * v)) / v=\max \left(D_{-}^{\sim} v+v\right)$, and if $\max (D * v)=D_{2}$ then $\max (D * v)$ and $(\max (D * v)) / v=(\max (D * v) / v) \oplus U_{1,1}(\{v\})$ are different matroids $(v$ is a coloop in the latter but not the former) with the same nullity.

\subsection{Representing graphs by $\Delta$-matroids}

Let $G=(V, E)$ be a graph. Recall Definition 28: $\mathcal{D}_{G}$ is the set system $(V, \sigma)$ where $\sigma=\{X \subseteq V \mid \mathcal{A}(G)[X]$ is nonsingular over $G F(2)\}$. It is shown in [9] that $\mathcal{D}_{G}$ is a normal $\Delta$-matroid (by convention, the empty matrix is nonsingular). Moreover, if $G$ is a looped simple graph then given $\mathcal{D}_{G}$, one can (re)construct $G:\{u\}$ is a loop in $G$ if and only if $\{u\} \in \mathcal{D}_{G}$, and $\{u, v\}$ is an edge in $G$ if and only if $\left(\{u, v\} \in \mathcal{D}_{G}\right) \Delta((\{u\} \in$ $\left.\left.\mathcal{D}_{G}\right) \wedge\left(\{v\} \in \mathcal{D}_{G}\right)\right)$, see [11, Property 3.1]. In this way, the family of looped simple graphs with vertex-set $V$ can be considered as a subset of the family of $\Delta$-matroids on the ground set $V$.

It is shown in [14, Theorem 6.2] that $d_{\mathcal{D}_{G}}(X)=\nu(\mathcal{A}(G)[X])$ for all $X \subseteq V$, where $\nu$ denotes $G F(2)$-nullity. If $v$ is a looped vertex of $G$, then it is shown in [20] that $\mathcal{D}_{G} * v$ represents the graph $G^{v}$. Moreover, if $v$ is an unlooped vertex of $G$, then $\mathcal{D}_{G} \bar{*} v$ represents the graph $G^{v}$ (see [13]). In this way, $G^{v}$ may be defined using $\Delta$-matroids. However, $\mathcal{D}_{G} * v$ on an unlooped vertex $v$ and $\mathcal{D}_{G} \bar{*} v$ on a looped vertex $v$ do not represent graphs in general:

Proposition 48 (Proof of Theorem 8.2 in [14]). Let $G$ be a graph, and $\varphi$ be any sequence of pivot, dual pivot and loop complement operations on elements of $V(G)$. Then $\varnothing \in$ $\left(\mathcal{D}_{G}\right) \varphi$ if and only if $\left(\mathcal{D}_{G}\right) \varphi=\mathcal{D}_{G^{\prime}}$ for some graph $G^{\prime}$.

In contrast, $\max \left(\left(\mathcal{D}_{G}\right) \varphi\right)$ does always have a graph representation.

Theorem 49. Let $G$ be a graph, and let $\varphi$ be any sequence of pivot, dual pivot and local complement operations on elements of $V(G)$. Then $\max \left(\left(\mathcal{D}_{G}\right) \varphi\right)$ is a binary matroid.

Proof. Let $D=\left(\mathcal{D}_{G}\right) \varphi$. Let $X \in \min (D)$. Then $\varnothing \in D \bar{*} X$ if and only if $\mid\{Z \in$ $D \mid Z \subseteq X\} \mid$ is odd. Since $\{Z \in D \mid Z \subseteq X\}=\{X\}$ by definition of $X$, we have $\varnothing \in D \bar{*} X=\left(\mathcal{D}_{G}\right) \varphi \bar{*} X$. By Proposition $48,\left(\mathcal{D}_{G}\right) \varphi \bar{*} X=\mathcal{D}_{G^{\prime}}$ for some graph $G^{\prime}$. Thus, $\max \left(\left(\mathcal{D}_{G}\right) \varphi\right)=\max \left(\left(\mathcal{D}_{G}\right) \varphi \bar{*} X\right)=\max \left(\mathcal{D}_{G^{\prime}}\right)$ and we are done.

For convenience, we define the pivot of a vertex $v$ on a graph $G$, denoted $G * v$, by $G^{v}$ if $v$ is looped, and it is not defined otherwise. Similarly, we define the dual pivot of vertex $v$ 
on $G$, denoted $G \bar{*} v$, by $G^{v}$ if $v$ is unlooped, and it is not defined otherwise. For a graph, loop complementation of a vertex $v \in V(G)$, denoted by $G+v$, toggles the existence of a loop on $v$. I.e., $v$ is a looped vertex of $G$ iff $v$ is not a looped vertex of $G+v$. It is shown in [13] that $\mathcal{D}_{G+v}=\mathcal{D}_{G}+v$ (i.e., loop complementation for $\Delta$-matroids generalizes loop complementation for graphs).

It is easy to verify that for each $v \in V(G), \mathcal{D}_{G-v}=\mathcal{D}_{G}-v$. Theorem 29 of the introduction follows readily from this easy observation and the strong principal minor theorem [24] (see also Theorem 40 above). As we will see in the following sections, the equality

$$
\max \left(\mathcal{D}_{G}\right)=M_{A}(G)
$$

(where $M_{A}(G)$ is described by its family of bases) allows us to give various results stated in the introduction completely different proofs, using $\Delta$-matroids rather than linear algebra over $G F(2)$.

\section{Deletion/contraction and $\min / \max$ for $\Delta$-matroids}

In this section we show, under the assumption of some mild conditions, that both contraction and deletion commute with both the min and the max operation for $\Delta$-matroids. In fact, some results hold for set systems in general. We will apply these results to graphs in the next section.

Let $D$ be a set system. The notions of loop and coloop for matroids (described by their families of bases) may be directly generalized to set systems. An element $v \in V$ is called a coloop of $D$ if $v \in X$ for each $X \in D$. Clearly, $v$ is a coloop of $D$ if and only if $D-v$ is not proper. Similarly, $v \in V$ is called a loop of $D$ if $v$ is a coloop of $D * v$, i.e., $v \notin X$ for each $X \in D$.

We first show that the min operation and the deletion operation on an element $v$ commute for proper set systems $D$, provided that $D-v$ is proper. Note that $D-v$ is proper, i.e., $v$ is not a coloop of $D$, if and only if $v$ is not a coloop of $\min (D)$.

Theorem 50. Let $D$ be a proper set system, and let $v \in V$ such that $D-v$ is proper. Then $\min (D)-v=\min (D-v)$.

Proof. Since $D-v$ is proper, $\min (D-v)$ is well defined. Let $X \in \min (D)-v$. Then $X \in \min (D)$ and $v \notin X$. Hence, $X \in \min (D-v)$. Conversely, if $X \in \min (D-v)$, then $X \in D$ and $v \notin X$. Let $Y \subseteq X$ with $Y \in \min (D)$. Then clearly, $v \notin Y$ and thus $Y \in \min (D-v)$. Hence $X=Y$ and $X \in \min (D)$. Therefore, $X \in \min (D)-v$.

Next, we show that the max operation and the deletion operation on a element $v$ commute for $\Delta$-matroids $D$, provided that $v$ is not a coloop of $\max (D)$.

Theorem 51. Let $D$ be a $\Delta$-matroid, and let $v \in V$ such that $v$ is not a coloop of $\max (D)$. Then $\max (D)-v=\max (D-v)$. 
Proof. Since $v$ is not a coloop of $\max (D)$, there is a $Z \in \max (D)$ with $v \notin Z$. Therefore $D-v$ is proper, and so $\max (D-v)$ is well defined. Let $X \in \max (D)-v$. Then $X \in \max (D)$ and $v \notin X$. Hence, $X \in \max (D-v)$. Conversely, if $X \in \max (D-v)$, then $X \in D$ and $v \notin X$, and $X$ is maximal with this property. As $v$ is not a coloop of $\max (D)$, there is a $Z \in \max (D)$ with $v \notin Z$. Therefore, $Z \in \max (D)-v$. By the first part of this proof, $Z \in \max (D-v)$. Now, as $D-v$ is a $\Delta$-matroid, $\max (D-v)$ is equicardinal and so $|Z|=|X|$. Moreover, since $D$ is a $\Delta$-matroid, $\max (D)$ is equicardinal, therefore $X \in \max (D)$ and so, $X \in \max (D)-v$.

The next example illustrates that Theorem 51 does not hold for set systems in general. This in contrast with Theorem 50, which does hold for set systems in general.

Example 52. Let $D=(V, \sigma)$ be a set system with $V=\{u, v, w\}$ and $\sigma=\{\{u\},\{v\}$, $\{v, w\}\}$. Then $w$ is not a coloop of $\max (D)=(V,\{\{u\},\{v, w\}\})$, and $\max (D)-w=$ $(\{u, v\},\{\{u\}\})$ while $\max (D-w)=(\{u, v\},\{\{u\},\{v\}\})$.

We formulate now the max (min, resp.) "counterparts" of Theorem 50 (Theorem 51, resp.). These results show that contraction commutes with the min and max operations.

Theorem 53. Let $D$ be a proper set system and $v \in V$.

1. If $v$ is not a loop of $D$, then $\max (D) * v-v=\max (D * v-v)$.

2. If $D$ is moreover a $\Delta$-matroid and $v$ is not a loop of $\min (D)$, then $\min (D) * v-v=$ $\min (D * v-v)$.

Proof. We start by showing the first result. We have $\max (D) * v-v=\min (D * V) * V *$ $v-v=\min (D * V)-v *(V \backslash\{v\})$. Now, $v$ is not a coloop of $D * V$. Thus, $D * V-v$ is proper. By Theorem 50, $\min (D * V)-v *(V \backslash\{v\})=\min (D * V-v) *(V \backslash\{v\})=$ $\min (D * v-v *(V \backslash\{v\})) *(V \backslash\{v\})=\max (D * v-v)$. The proof of the second result is essentially identical to that of the first result. We have $\min (D) * v-v=\max (D * V) * V *$ $v-v=\max (D * V)-v *(V \backslash\{v\})$. Now, $v$ is not a coloop of $\min (D) * V=\max (D * V)$. By Theorem 51, $\max (D * V)-v *(V \backslash\{v\})=\max (D * V-v) *(V \backslash\{v\})=\max (D * v-$ $v *(V \backslash\{v\})) *(V \backslash\{v\})=\min (D * v-v)$.

\section{From $\Delta$-matroids to graphs}

In this section we use results of Sections 9 and 10 to give new proofs of several theorems about adjacency matroids stated earlier in the paper. These proofs are fundamentally different from the earlier ones, as they are combinatorial and do not involve matrices. Recall that as observed in Subsection 9.3, if $G$ is a graph then $\mathcal{D}_{G}$ is a $\Delta$-matroid with $M_{A}(G)=\max \left(\mathcal{D}_{G}\right) ; \mathcal{D}_{G}$ is normal, so no $v \in V$ is a coloop of $\mathcal{D}_{G}$.

The following three results are quite straightforward consequences of the fact that for $\mathcal{D}_{G}$, max commutes with both deletion (of non-coloops) and contraction (of non-loops), cf. Theorems 51 and 53 . 
Theorem 54. If $v$ is not a coloop of $M_{A}(G)$, then $M_{A}(G)-v=M_{A}(G-v)$.

Proof. If $v$ is not a coloop of $M_{A}(G)$, then $M_{A}(G)-v=\max \left(\mathcal{D}_{G}\right)-v$. By Theorem 51, $\max \left(\mathcal{D}_{G}\right)-v=\max \left(\mathcal{D}_{G}-v\right)=\max \left(\mathcal{D}_{G-v}\right)=M_{A}(G-v)$.

Theorem 55. If $v \in V(G)$ is a looped vertex, then $M_{A}(G) / v=M_{A}\left(G^{v}-v\right)$.

Proof. Since $\{v\} \in \mathcal{D}_{G}, v$ is not a loop of $\mathcal{D}_{G}$. Consequently, $v$ is not a loop of $\max \left(\mathcal{D}_{G}\right)$. We have therefore $M_{A}(G) / v=\max \left(\mathcal{D}_{G}\right) * v-v$. By Theorem 53, $\max \left(\mathcal{D}_{G}\right) * v-v=$ $\max \left(\mathcal{D}_{G} * v-v\right)$. Since $v$ is a looped vertex, $\mathcal{D}_{G} * v=\mathcal{D}_{G * v}$, and thus $\max \left(\mathcal{D}_{G} * v-v\right)=$ $\max \left(\mathcal{D}_{G * v-v}\right)=M_{A}(G * v-v)$. The result follows as $G * v=G^{v}$.

Theorem 56. Suppose $v$ is an unlooped vertex of $G$.

1. If $v$ is isolated, then $M_{A}(G) / v=M_{A}(G-v)$.

2. If $w$ is an unlooped neighbor of $v$, then $M_{A}(G) / v=M_{A}\left(\left(G^{w}\right)^{v}-v\right)$.

3. If $w$ is a looped neighbor of $v$, then $M_{A}(G) / v=M_{A}\left(\left(\left(G^{v}\right)^{w}\right)^{v}-v\right)$.

Proof. We first prove Result 1. If $v$ is isolated and unlooped, then $v$ is a loop of $M_{A}(G)$. Hence, $M_{A}(G) / v=M_{A}(G)-v$. Moreover, $v$ is not a coloop of $M_{A}(G)$. The result follows now by Theorem 54 .

We now prove Results 2 and 3. Let $w$ be a neighbor of $v$. As $\{v, w\} \in \mathcal{D}_{G}, v$ is not a loop of $\mathcal{D}_{G}$. Hence, $M_{A}(G) / v=\max \left(\mathcal{D}_{G}\right) * v-v$. By Theorem $53, \max \left(\mathcal{D}_{G}\right) * v-v=$ $\max \left(\mathcal{D}_{G} * v-v\right)$. Now, $\max \left(\mathcal{D}_{G} * v-v\right)=\max \left(\mathcal{D}_{G} * v-v \bar{*} w\right)=\max \left(\mathcal{D}_{G} \bar{*} w * v-v\right)$. On the one hand, if $w$ is unlooped, then it is easy to verify that $G \bar{*} w * v$ is defined. Hence $\mathcal{D}_{G} \bar{*} w * v=\mathcal{D}_{G \bar{*} w * v}$. Finally, $\max \left(\mathcal{D}_{G \bar{*} w * v}-v\right)=M_{A}(G \bar{*} w * v-v)=M_{A}\left(\left(G^{w}\right)^{v}-v\right)$. This proves Result 2. On the other hand, if $w$ is looped, then it is easy to verify that

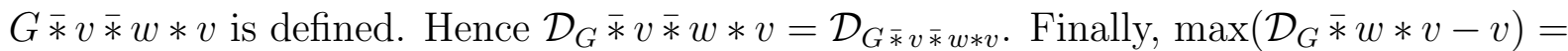
$\max \left(\mathcal{D}_{G \bar{*} v \bar{*} w * v}-v\right)=M_{A}(G \bar{*} v \bar{*} w * v-v)=M_{A}\left(\left(\left(G^{v}\right)^{w}\right)^{v}-v\right)$. This proves Result 3.

The next result is obtained from Proposition 47.

Theorem 57. 1. If $v \in V(G)$ is unlooped, then $M_{A}\left(G^{v}\right)=M_{A}(G)$.

2. If $v \in V(G)$ is a coloop of both $M_{A}(G)$ and $M_{A}\left(G^{v}\right)$, then $M_{A}\left(G^{v}\right)=M_{A}(G)$.

3. If $v \in V(G)$ is looped and not a coloop of one of $M_{A}(G), M_{A}\left(G^{v}\right)$, then $v$ is a coloop of the other and $M_{A}\left(G^{v}\right)$ and $M_{A}(G)$ are of different ranks.

Proof. We first show Result 1. If $v$ is unlooped, then $G^{v}=G \bar{*} v$. Thus, $M_{A}(G)=$ $\max \left(\mathcal{D}_{G}\right)=\max \left(\mathcal{D}_{G} \bar{*} v\right)=\max \left(\mathcal{D}_{G \bar{*} v}\right)=M_{A}\left(G^{v}\right)$ and the result follows.

We now show Results 2 and 3. If $v$ is unlooped, then we are done by Result 1 . So, assume $v$ is looped. Then we have $G^{v}=G * v$. The result follows now by Proposition 47 .

Theorem 58. If $v \in V(G)$, then $M_{A}(G)-v=M_{A}\left(G^{v}\right)-v$. 
Proof. If $v$ is an unlooped vertex, then by Theorem 57.1, $M_{A}(G)=M_{A}\left(G^{v}\right)$ and the equality holds. Assume now that $v$ is a looped vertex. By Theorem 57.3, $v$ is a coloop of at least one of $M_{A}(G)$ and $M_{A}\left(G^{v}\right)$. If $v$ is a coloop of both $M_{A}(G)$ and $M_{A}\left(G^{v}\right)$, then the equality holds by Theorem 57.2 .

We assume now without loss of generality that $v$ is a coloop of $M_{A}(G)$ and $v$ is not a coloop of $M_{A}\left(G^{v}\right)$ (the other case follows by considering graph $G:=G^{v}$ ). We have in this case $M_{A}(G)-v=M_{A}(G) / v$. By Theorem 55, $M_{A}(G) / v=M_{A}\left(G^{v}-v\right)$. As $v$ is not a coloop of $M_{A}\left(G^{v}\right)$, by Theorem 54, $M_{A}\left(G^{v}-v\right)=M_{A}\left(G^{v}\right)-v$ and the result follows.

By the way, the interested reader will have no trouble using [14, Theorem 6.2] to prove Theorems 44 and 45.

It is easy to see that $\mathcal{D}_{G} \stackrel{\sim}{-} v+v=\mathcal{D}_{G(v, l i)}$. Hence we obtain the following corollary to Theorem 46 and Proposition 47. Part 1 follows from part 2, which is part of Proposition 24; and part 3 includes some of the assertions of Theorem 25.

Theorem 59. If $G$ is a graph with a looped vertex $v$, then the following hold.

1. $\nu\left(M_{A}\left(G^{v}\right)\right)=\nu\left(M_{A}(G(v, \ell i))\right)$.

2. $\mathcal{B}\left(M_{A}(G(v, \ell i))\right)=\left\{B \in \mathcal{B}\left(M_{A}\left(G^{v}\right)\right) \mid v \in B\right\}$, i.e., $M_{A}(G(v, \ell i))=\left(M_{A}\left(G^{v}\right) / v\right) \oplus$ $U_{1,1}(\{v\})$.

3. $M_{A}(G(v, \ell i))=M_{A}\left(G^{v}\right)$ iff $\nu\left(M_{A}(G)\right) \geqslant \nu\left(M_{A}\left(G^{v}\right)\right)$.

\section{The interlace and Tutte polynomials}

In this section we discuss the connection between the interlace polynomials of a graph $G$, introduced by Arratia, Bollobás and Sorkin [2, 3, 4], and the Tutte polynomials of the adjacency matroids of $G$ and its subgraphs. (The Tutte polynomial is described by many authors; see [6] and [34] for instance. Especially thorough accounts are given in the book chapters by Brylawski and Oxley [38] and Ellis-Monaghan and Merino [17].) In particular, we show that the fundamental recursion of the two-variable interlace polynomial may be derived from properties of the leading term of the Tutte polynomial.

Definition 60. Let $G$ be a graph. Then the interlace polynomial of $G$ is

$$
\begin{aligned}
q(G) & =\sum_{S \subseteq V(G)}(x-1)^{|S|-\nu(\mathcal{A}(G[S]))}(y-1)^{\nu(\mathcal{A}(G[S]))} \\
& =\sum_{S \subseteq V(G)}(x-1)^{|S|} \cdot\left(\frac{y-1}{x-1}\right)^{\nu(\mathcal{A}(G[S]))},
\end{aligned}
$$

where $\nu$ denotes $G F(2)$-nullity. 
Arratia, Bollobás and Sorkin [4] showed that $q(G)$ may also be defined recursively:

1. If $v$ is a looped vertex of $G$ then $q(G)=q(G-v)+(x-1) q\left(G^{v}-v\right)$.

2. If $v$ and $w$ are unlooped neighbors in $G$ then $q(G)=q(G-v)+q\left(\left(\left(G^{v}\right)^{w}\right)^{v}-v\right)$ $+\left((x-1)^{2}-1\right) \cdot q\left(\left(\left(G^{v}\right)^{w}\right)^{v}-v-w\right)$.

3. If $G$ consists solely of unlooped vertices then $q(G)=y^{|V(G)|}$.

So far, our discussion of matroids has been focused on their circuits. Here are two other basic definitions of matroid theory.

Definition 61. Let $M$ be a matroid on a set $V$. A subset $I \subseteq V$ is independent if $I$ contains no circuit of $M$. The rank of a subset $S \subseteq V$ is the cardinality of the largest independent set(s) in $S$; it is denoted $r(S)$.

All the notions of matroid theory can be equivalently defined from the independent sets or the rank function, instead of the circuits. For instance, Definitions 15 and 16 are equivalent to: if $M$ is a matroid on a set $V$ and $v \in V$ then the deletion $M-v$ and the contraction $M / v$ are the matroids on $V \backslash\{v\}$ with the rank functions $r_{M-v}(S)=r(S)$ and $r_{M / v}(S)=r(S \cup\{v\})-r(\{v\})$.

Recall that if $G$ is a graph, then the circuits of $M_{A}(G)$ are the minimal nonempty subsets $S \subseteq V(G)$ such that the columns of $\mathcal{A}(G)$ corresponding to elements of $S$ are linearly dependent. It follows that the rank in $M_{A}(G)$ of a subset $S \subseteq V(G)$ is simply the $G F(2)$-rank of the $|V(G)| \times|S|$ submatrix of $\mathcal{A}(G)$ obtained by removing the columns corresponding to vertices not in $S$. This submatrix of $\mathcal{A}(G)$ is obtained from $\mathcal{A}(G[S])$ by adjoining rows corresponding to vertices not in $S$, so $r(S) \geqslant|S|-\nu(\mathcal{A}(G[S]))$. The difference between $r(S)$ and $|S|-\nu(\mathcal{A}(G[S]))$ varies with $G$ and $S$, in general; however if $r(S)=r\left(M_{A}(G)\right)$ then according to the strong principal minor theorem (see [24] or Theorem 40), $r(S)=|S|-\nu(\mathcal{A}(G[S]))$.

Definition 62. Let $M$ be a matroid on a set $V$. Then the Tutte polynomial of $M$ is

$$
t(M)=\sum_{S \subseteq V}(x-1)^{r(V)-r(S)}(y-1)^{|S|-r(S)} .
$$

The equations $r_{M / v}(S)=r(S \cup\{v\})-r(\{v\})$ and $r_{M-v}(S)=r(S)$ imply that the Tutte polynomial may be calculated recursively using the following steps.

1. If $v$ is a loop of $M$ then $t(M)=y \cdot t(M-v)$.

2. If $v$ is a coloop of $M$ then $t(M)=x \cdot t(M / v)$.

3. If $v$ is neither a loop nor a coloop, then $t(M)=t(M / v)+t(M-v)$.

4. $t(\varnothing)=1$.

The distinction between $M-v$ and $M / v$ in steps 1 and 2 is traditional, but for us it is unimportant as Definitions 15 and 16 have $M / v=M-v$ for loops and coloops.

We single out the leading term of $t(M)$ (the term corresponding to $S=V$ ) for special attention.

Definition 63. Let $M$ be a matroid on a set $V$. Then $\lambda_{M}(y)=(y-1)^{|V|-r(V)}$. 
Like $t(M), \lambda_{M}$ has a recursive description derived from the equations $r_{M / v}(S)=$ $r(S \cup\{v\})-r(\{v\})$ and $r_{M-v}(S)=r(S)$ :

Proposition 64. 1. If $v$ is a loop of $M$ then $\lambda_{M}=(y-1) \cdot \lambda_{M-v}=(y-1) \cdot \lambda_{M / v}$.

2. If $v$ is a coloop of $M$ then $\lambda_{M}=\lambda_{M-v}=\lambda_{M / v}$.

3. If $v$ is neither a loop nor a coloop, then $\lambda_{M}=(y-1) \cdot \lambda_{M-v}=\lambda_{M / v}$.

4. $\lambda_{\varnothing}=1$.

If $G$ is a graph then we adopt the abbreviated notation $\lambda_{M_{A}(G)}=\lambda_{G}$.

Corollary 65. 1. If $v$ is an unlooped vertex of $G$ then $\lambda_{G}=\lambda_{G^{v}}$.

2. If $v$ is a looped vertex of $G$ then $\lambda_{G}=\lambda_{G^{v}-v}$.

3. If $v$ is an isolated, unlooped vertex of $G$ then $\lambda_{G}=(y-1) \cdot \lambda_{G-v}$.

4. $\lambda_{\varnothing}=1$.

Proof. If $v$ is an unlooped vertex of $G$ then $M_{A}(G)=M_{A}\left(G^{v}\right)$ by Theorem 21. If $v$ is a looped vertex of $G$ then $v$ is not a loop of $M_{A}(G)$, so $\lambda_{M_{A}(G)}=\lambda_{M_{A}(G) / v}=\lambda_{G^{v}-v}$ by Proposition 64 and Theorem 17. If $v$ is an isolated, unlooped vertex of $G$ then $v$ is a loop of $M_{A}(G)$, so $\lambda_{M_{A}(G)}=(y-1) \cdot \lambda_{M_{A}(G)-v}=(y-1) \cdot \lambda_{G-v}$ by Theorem 19 and Proposition 64 .

If $G$ is a graph then Definitions 60 and 63 imply that

$$
q(G)=\sum_{S \subseteq V(G)}(x-1)^{|S|} \cdot \lambda_{G[S]}\left(1+\frac{y-1}{x-1}\right)
$$

and hence for each $v \in V(G)$,

$$
q(G)-q(G-v)=\sum_{v \in S \subseteq V(G)}(x-1)^{|S|} \cdot \lambda_{G[S]}\left(1+\frac{y-1}{x-1}\right) .
$$

Suppose $v$ is a looped vertex and $v \in S \subseteq V(G)$. Then Corollary 65 tells us that $\lambda_{G[S]}$ $=\lambda_{G[S]^{v}-v}$. Clearly $G[S]^{v}-v=G^{v}[S]-v=\left(G^{v}-v\right)[S \backslash\{v\}]$, so it follows from equations (1) and (2) that

$$
\begin{aligned}
q(G)-q(G-v) & =(x-1) \cdot \sum_{v \in S \subseteq V(G)}(x-1)^{|S|-1} \cdot \lambda_{G^{v}[S]-v}\left(1+\frac{y-1}{x-1}\right) \\
& =(x-1) \cdot \sum_{S \subseteq V(G) \backslash\{v\}}(x-1)^{|S|} \cdot \lambda_{\left(G^{v}-v\right)[S]}\left(1+\frac{y-1}{x-1}\right) \\
& =(x-1) \cdot q\left(G^{v}-v\right) .
\end{aligned}
$$

This yields the first formula of the recursive description of $q$.

Suppose now that $v$ is an unlooped vertex of $G$, and $w$ is an unlooped neighbor of $v$ in $G$. Let $H=\left(G^{v}\right)^{w}$; then $v$ and $w$ are looped neighbors in $H$. Equation (2) tells us that

$$
q\left(H^{v}-v\right)-q\left(H^{v}-v-w\right)=\sum_{w \in S \subseteq V\left(H^{v}-v\right)}(x-1)^{|S|} \cdot \lambda_{H^{v}[S]}\left(1+\frac{y-1}{x-1}\right) .
$$


Suppose $w \in S \subseteq V\left(H^{v}-v\right)$; obviously then $H^{v}[S]=H^{v}[S \cup\{v\}]-v=H[S \cup\{v\}]^{v}-v$. As $v$ and $w$ are both looped in $H[S \cup\{v\}]=\left(G^{v}\right)^{w}[S \cup\{v\}]$, Corollary 65 tells us that

$$
\lambda_{H^{v}[S]}=\lambda_{H[S \cup\{v\}]^{v}-v}=\lambda_{H[S \cup\{v\}]}=\lambda_{\left(G^{v}\right)^{w}[S \cup\{v\}]}=\lambda_{\left(\left(G^{v}\right)^{w}[S \cup\{v\}]\right)^{w}-w} .
$$

Note that $\left(\left(G^{v}\right)^{w}[S \cup\{v\}]\right)^{w}-w=\left(\left(G^{v}\right)^{w}\right)^{w}[S \cup\{v\}]-w=G^{v}[(S \backslash\{w\}) \cup\{v\}]=$ $G[(S \backslash\{w\}) \cup\{v\}]^{v}$. As $v$ is unlooped in $G[(S \backslash\{w\}) \cup\{v\}]$, Corollary 65 tells us that

$$
\lambda_{\left(\left(G^{v}\right)^{w}[S \cup\{v\}]\right)^{w}-w}=\lambda_{G[(S \backslash\{w\}) \cup\{v\}]^{v}}=\lambda_{G[(S \backslash\{w\}) \cup\{v\}] .} .
$$

We conclude that

$$
\begin{aligned}
& q\left(H^{v}-v\right)-q\left(H^{v}-v-w\right) \\
& =\sum_{w \in S \subseteq V(H-v)}(x-1)^{|S|} \cdot \lambda_{G[(S \backslash\{w\}) \cup\{v\}]}\left(1+\frac{y-1}{x-1}\right) \\
& =\sum_{v \in S \subseteq V(G-w)}(x-1)^{|S|} \cdot \lambda_{G[S]}\left(1+\frac{y-1}{x-1}\right) .
\end{aligned}
$$

Combining this with equation (2), we see that

$$
\begin{aligned}
& q(G)-q(G-v) \\
& =q\left(H^{v}-v\right)-q\left(H^{v}-v-w\right)+\sum_{v, w \in S \subseteq V(G)}(x-1)^{|S|} \cdot \lambda_{G[S]}\left(1+\frac{y-1}{x-1}\right) .
\end{aligned}
$$

Suppose now that $v, w \in S \subseteq V(G)$. Then Proposition 64 and Theorem 18 imply that

$$
\lambda_{G[S]}=\lambda_{M_{A}(G[S]) / w}=\lambda_{\left(G[S]^{v}\right)^{w}-w}=\lambda_{\left(G^{v}\right)^{w}[S]-w}=\lambda_{H[S \backslash\{w\}] .}
$$

As $v$ is looped in $H$, Corollary 65 states that

$$
\lambda_{H[S \backslash\{w\}]}=\lambda_{H[S \backslash\{w\}]^{v}-v}=\lambda_{H^{v}[S \backslash\{v, w\}]} .
$$

We conclude that

$$
\begin{aligned}
& q(G)-q(G-v)-q\left(H^{v}-v\right)+q\left(H^{v}-v-w\right) \\
& =\sum_{v, w \in S \subseteq V(G)}(x-1)^{|S|} \cdot \lambda_{G[S]}\left(1+\frac{y-1}{x-1}\right) \\
& =\sum_{v, w \in S \subseteq V(G)}(x-1)^{|S|} \cdot \lambda_{H^{v}[S \backslash\{v, w\}]}\left(1+\frac{y-1}{x-1}\right) \\
& =\sum_{S \subseteq V\left(H^{v}-v-w\right)}(x-1)^{|S|+2} \cdot \lambda_{H^{v}[S]}\left(1+\frac{y-1}{x-1}\right) \\
& =(x-1)^{2} \cdot q\left(H^{v}-v-w\right) .
\end{aligned}
$$


This yields the second formula of the recursive description of $q$.

In the years since Arratia, Bollobás and Sorkin introduced the interlace polynomials $[2,3,4]$, several related graph polynomials have been studied by other researchers $[1,16$, 32]. These related polynomials have definitions similar to Definition 60, as sums involving $G F(2)$-nullities of symmetric matrices. Consequently they have similar connections with the leading terms of Tutte polynomials of adjacency matroids.

\section{References}

[1] M. Aigner and H. van der Holst. Interlace polynomials. Linear Algebra Appl., 377:1130, 2004.

[2] R. Arratia, B. Bollobás, and G. B. Sorkin. The interlace polynomial: A new graph polynomial. In Proceedings of the Eleventh Annual ACM-SIAM Symposium on Discrete Algorithms (San Francisco, 2000), pages 237-245. Association for Computing Machinery, New York, 2000.

[3] R. Arratia, B. Bollobás, and G. B. Sorkin. The interlace polynomial of a graph. J. Combinatorial Theory Ser. B, 92:199-233, 2004.

[4] R. Arratia, B. Bollobás, and G. B. Sorkin. A two-variable interlace polynomial. Combinatorica, 24:567-584, 2004.

[5] P. N. Balister, B. Bollobás, J. Cutler, and L. Pebody. The interlace polynomial of graphs at -1. Europ. J. Combinatorics, 23:761-767, 2002.

[6] B. Bollobás. Modern Graph Theory. Springer-Verlag, New York, 1998.

[7] A. Bouchet. Caractérisation des symboles croisés de genre nul. C. R. Acad. Sci. Paris Sér. A-B, 274:A724-A727, 1972.

[8] A. Bouchet. Isotropic systems. Europ. J. Combinatorics, 8:231-244, 1987.

[9] A. Bouchet. Representability of $\Delta$-matroids. In Combinatorics (Eger, 1987), volume 52 of Colloq. Math. Soc. János Bolyai, pages 167-182. North-Holland, Amsterdam, 1988.

[10] A. Bouchet. Coverings and delta-coverings. In Integer Programming and Combinatorial Optimization (Copenhagen, 1995), volume 920 of Lecture Notes in Comput. Sci., pages 228-243. Springer, Berlin, 1995.

[11] A. Bouchet and M. Duchamp. Representability of $\Delta$-matroids over $G F(2)$. Linear Algebra Appl., 146:67-78, 1991.

[12] H. R. Brahana. Systems of circuits on two-dimensional manifolds. Ann. Math., 23:144-168, 1921.

[13] R. Brijder and H. J. Hoogeboom. The group structure of pivot and loop complementation on graphs and set systems. Europ. J. Combinatorics, 32:1353-1367, 2011.

[14] R. Brijder and H. J. Hoogeboom. Nullity and loop complementation for deltamatroids. SIAM J. Discrete Math., 27:492-506, 2013. 
[15] R. Brijder and H. J. Hoogeboom. Interlace polynomials for delta-matroids. preprint, arXiv:1010.4678.

[16] B. Courcelle. A multivariate interlace polynomial and its computation for graphs of bounded clique-width. Electron. J. Combin., 15\#R69, 2008.

[17] M. Dehmer, ed. Structural Analysis of Complex Networks. Birkhäuser, Boston, 2011.

[18] J. A. Ellis-Monaghan and I. Sarmiento. Distance hereditary graphs and the interlace polynomial. Combin. Prob. Comput., 16:947-973, 2007.

[19] A. Ghouila-Houri. Flots et tensions dans un graphe. Ann. Scient. Ec. Norm. Sup., 81:267-339, 1964.

[20] J. F. Geelen. A generalization of Tutte's characterization of totally unimodular matrices. J. Combinatorial Theory Ser. B, 70:101-117, 1997.

[21] F. Jaeger. Symmetric representations of binary matroids. Ann. Discrete Math., 17:371-376, 1983.

[22] F. Jaeger. Graphes de cordes et espaces graphiques. Europ. J. Combinatorics, 4:319327, 1983.

[23] F. Jaeger. On some algebraic properties of graphs. In Progress in Graph Theory (Waterloo, 1982), pages 347-366. Academic Press, Toronto, 1984.

[24] V. Kodiyalam, T. Y. Lam, and R. G. Swan. Determinantal ideals, Pfaffian ideals, and the principal minor theorem. In Noncommutative Rings, Group Rings, Diagram Algebras and Their Applications, volume 456 of Contemp. Math., pages 35-60. Amer. Math. Soc., Providence, 2008.

[25] A. Kotzig. Eulerian lines in finite 4-valent graphs and their transformations. In Theory of Graphs (Proc. Colloq., Tihany, 1966), pages 219-230. Academic Press, New York, 1968.

[26] J. G. Oxley. Matroid Theory. Oxford Univ. Press, Oxford, 2011.

[27] P. Rosenstiehl and R. C. Read. On the Gauss crossing problem. In Combinatorics (Proc. Fifth Hungarian Colloq., Keszthely, 1976), Vol. II, volume 18 of Colloq. Math. Soc. János Bolyai, pages 843-876. North-Holland, Amsterdam-New York, 1978.

[28] P. Rosenstiehl and R. C. Read. On the principal edge tripartition of a graph. Ann. Disc. Math., 3:195-226, 1978.

[29] L. Traldi. Weighted interlace polynomials. Combin. Probab. Comput., 19:133-157, 2010.

[30] L. Traldi. Binary nullity, Euler circuits and interlacement polynomials. Europ. J. Combinatorics, 32:944-950, 2011.

[31] L. Traldi. On the linear algebra of local complementation. Linear Algebra Appl., 436:1072-1089, 2012.

[32] L. Traldi. On the interlace polynomials. J. Combinatorial Theory Ser. B, 103:184208, 2013. 
[33] L. Traldi. Interlacement in 4-regular graphs: a new approach using nonsymmetric matrices. preprint, arXiv:1204.0482.

[34] W. T. Tutte. Graph Theory. Cambridge Univ. Press, Cambridge, 1984.

[35] D. J. A. Welsh. Matroid Theory. Academic Press, London, 1976.

[36] N. White, ed. Theory of Matroids. Cambridge Univ. Press, Cambridge, 1986.

[37] N. White, ed. Combinatorial Geometries. Cambridge Univ. Press, Cambridge, 1987.

[38] N. White, ed. Matroid Applications. Cambridge Univ. Press, Cambridge, 1992. 